\title{
Meteoroids as One of the Sources for Exosphere Formation on Airless Bodies in the Inner Solar System
}

\author{
Diego Janches ${ }^{1}$ - Alexey A. Berezhnoy ${ }^{2,3}$. Apostolos A. Christou ${ }^{4}$. \\ Gabriele Cremonese $^{5}$. Takayuki Hirai ${ }^{6}$. Mihály Horányi ${ }^{7,8,9}$. Jamie M. Jasinski ${ }^{10}$. \\ Menelaos Sarantos ${ }^{11}$
}

Received: 7 October 2020 / Accepted: 19 March 2021 / Published online: 19 April 2021

(c) The Author(s) 2021

\begin{abstract}
This manuscript represents a review on progress made over the past decade concerning our understanding of meteoroid bombardment on airless solar system bodies as one of the sources of the formation of their exospheres. Specifically, observations at Mercury by MESSENGER and at the Moon by LADEE, together with progress made in dynamical models of the meteoroid environment in the inner solar system, offer new tools to explore in detail the physical phenomena involved in this complex relationship. This progress is timely given the expected results during the next decade that will be provided by new missions such as DESTINY $^{+}$, BepiColombo, the Artemis program or the Lunar Gateway.
\end{abstract}

Keywords Meteoroids $\cdot$ Meteoroid streams $\cdot$ Exospheres $\cdot$ Mercury $\cdot$ Moon

\section{Introduction}

The influence of interplanetary dust on planetary bodies is an ubiquitous phenomenon in the solar system. Planets, moons and asteroids sweep through a cloud of these particles while they move along their orbital path causing a constant bombardment of meteoroids on their surfaces and atmospheres. At planetary atmospheres, meteoroids heat up as they interact with an increasingly denser atmosphere while decelerating. If they are sufficiently large ( $>30 \mu \mathrm{m}$ in radius) they will ablate most of their material in the atmospheric aerobraking region, introducing exotic species such as $\mathrm{Mg}, \mathrm{Fe}$, and $\mathrm{Na}$ into the atmosphere (CarrilloSánchez et al. 2020; Crismani et al. 2018, 2017; Grebowsky et al. 2002, 2017; Plane 2003; Berezhnoy and Borovička 2010). For this case, the ablation processes will result in ionization and/or photon production, generating the well known meteor phenomenon (Ceplecha et al. 1998). As these metallic species can be ionized during ablation either by solar ultraviolet photoionization or by charge exchange with existing atmospheric ions, meteoroids therefore affect the structure, chemistry, dynamics, and energetics of planetary ionospheres (Plane et al. 2015, 2018; Plane 2003).

Surface-Bounded Exospheres and Interactions in the Inner Solar System

Edited by Anna Milillo, Menelaos Sarantos, Benjamin D. Teolis, Go Murakami, Peter Wurz and Rudolf von Steiger

Extended author information available on the last page of the article 
For the case of airless bodies, meteoroids impact their surfaces directly, producing impact debris and directly shaping the resulting thin exospheres. Several works have shown direct observational evidence of the critical role that meteoroid activity plays in space weathering of airless bodies using observations from NASA missions such as the MErcury Surface, Space ENvironment, GEochemistry, and Ranging (MESSENGER) mission and the Lunar Atmosphere and Dust Environment Explorer (LADEE) (Janches et al. 2018; Pieters and Noble 2016; Pokorný et al. 2019, 2017b, 2018; Szalay et al. 2018). Therefore, knowledge of the meteoroid environment is relevant to planetary science, chemistry of planetary atmospheres, space weathering of airless bodies and even collisional risk assessment to artificial satellites and astronauts (Moorhead et al. 2020).

Recently, Plane et al. (2018) reported a detailed review on the impact and effects of meteoroids in planetary atmospheres. Hence, the principal scientific goal of this review article is to summarize our current knowledge of the role of meteoroids and dust as one of the sources of the hermean and lunar exospheres. A summary of current dynamical models of the most relevant particle populations are presented as well as current observational methods, including both ground-based and satellite observations, and descriptions of laboratory experiments designed to study meteoroids as a source of exospheric formation. Although this review article mostly focuses on the two aforementioned airless bodies, consideration of additional bodies (i.e. asteroids) is also briefly discussed.

For the remainder of this review article, we will adopt the nomenclature used in meteor astronomy that was approved in 2016 by the International Astronomical Union (IAU) Commission F1: Meteors, Meteoroids and Interplanetary Dust. In that respect, a meteoroid is defined as a solid natural object of a size roughly between $30 \mu \mathrm{m}$ and $1 \mathrm{~m}$ moving in, or coming from, interplanetary space. Dust (interplanetary) is finely divided solid matter, with particle sizes generally smaller than meteoroids, moving in, or coming from, interplanetary space. Dust in the solar system is observed e.g. as the zodiacal dust cloud (ZDC), including zodiacal dust bands, and cometary dust trails. In such contexts, the term "dust" also includes smaller meteoroids; i.e., the ZDC and cometary dust trails contain larger particles that can also be called meteoroids. As implied above, small dust particles do not give rise to the meteor phenomenon when they enter planetary atmospheres. A fraction of these particles will be heated to below the melting point and will subsequently sediment to the ground. These unmelted particles can still hold signatures from their atmospheric entry (e.g. vesicles, loss of volatile elements). When collected on the ground, they are called micrometeorites. When collected in the atmosphere, they are called interplanetary dust particles (IDP's). When in interplanetary space, they are simply called dust particles. Particularly relevant to the topic of this review article is that foreign objects on the surfaces of atmosphere-less bodies are not called meteorites (i.e. there is no meteorite without a meteor) and so they are referred to as impact debris.

\section{Meteoroid Populations in the Near Earth Environment: Observations and Models}

Observing meteors is most likely an activity practiced by humanity since its beginnings, and thus it is natural to start describing the meteoroid environment with what we have learned from Earth-based meteor studies. Meteoroids that produce meteors bright enough to be seen with the naked eye are, in general, larger than the mass range of interest to this chapter. The number of meteors impinging on a solar system body is inversely proportional to their size in a log-log scale (see for example Fig. 25 in Ceplecha et al. 1998). In terms of a 
Table 1 In-situ dust measurements in interplanetary space (with updates to Grün et al. (2001))

\begin{tabular}{llll}
\hline Spacecraft & Distance range [AU] & Sensitive area $\left[\mathrm{m}^{2}\right]$ & Reference \\
\hline Helios 1/2 & $0.3-1$ & 0.012 & Dietzel et al. (1973) \\
Galileo & $0.7-5.4$ & 0.1 & Grün et al. (1992) \\
Cassini & $0.72-10$ & 0.1 & Srama et al. (2004) \\
IKAROS & $0.72-1.1$ & 0.54 & Hirai et al. (2017) \\
Pioneer 9 & $0.75-0.99$ & 0.0074 & Rhee et al. (1974) \\
Pioneer 8 & $0.97-1.09$ & 0.0094 & Berg and Richardson (1969) \\
Explorer XVI & 1 & 1.6 & Hastings (1963) \\
Explorer XXIII & 1 & 2.1 & O'Neal et al. (1965) \\
Pegasus & 1 & 200 & D'Aiutolo et al. (1967) \\
Hiten & 1 & 0.01 & Igenbergs et al. (1991) \\
HEOS 2 & 1 & 0.01 & Dietzel et al. (1973) \\
Nozomi & $1-1.5$ & 0.014 & Sasaki et al. (2007) \\
Mariner IV & $1-1.56$ & 0.048 & Alexander et al. (1967) \\
Ulysses & $1-5.4$ & 0.1 & Grün et al. (1992) \\
Pioneer 10 & $1-18$ & $0.26^{\mathrm{a}}$ & Humes (1980) \\
Pioneer 11 & $1-10$ & $0.56^{\mathrm{a}}$ & Humes (1980) \\
LADEE & 1 & 0.01 & Horányi et al. (2014) \\
New Horizons & $1-48^{\mathrm{b}}$ & 0.1 & Horányi et al. (2008) \\
\hline & & &
\end{tabular}

ainitial area, actual area decreased as the pressurized cells were punctured

$b_{\text {as of }} 6 / 2020$

source capable of producing and maintaining a planetary exosphere, we are interested in a size (or mass) range of particles that are "large" enough to produce sufficient energy to vaporize enough mass from the surface of the meteoroid after colliding with the surface of the airless body and, oftentimes, may trigger a continuous source of collisions. It is widely accepted that this "sweet spot" covers meteoroid masses in the range of 0.1 to $1000 \mu \mathrm{g}$ with a peak of the flux that varies somewhat depending on the reported work. For example, Fig. 5 in Carrillo-Sánchez et al. (2015) shows that the LDEF mass distribution peaks at $50 \mu \mathrm{g}$ (around $400 \mu \mathrm{m}$ in diameter assuming a bulk density of $2.2 \mathrm{~g} / \mathrm{cm}^{3}$ and an impact velocity of $18 \mathrm{~km} \mathrm{~s}^{-1}$ ). However, the Zodiacal Cloud Model constrained by the Planck observations Ade et al. (2014) clearly shows that the average diameter range from $0.01 \mu \mathrm{g}$ (30 $\mu \mathrm{m}$, JFCs) to $50 \mu \mathrm{g}(400 \mu \mathrm{m}, \mathrm{HTCs})$.

At Earth, the flux of particles in this mass range have been observed with space-borne detectors such as the Long Duration Exposure Facility (LDEF) (Love and Brownlee 1993; Love and Allton 2006; Miao and Stark 2001; Cremonese et al. 2012), and the Cosmic Dust Experiment onboard the Aeronomy of Ice in the Mesosphere (AIM) mission (Russell et al. 2009; Poppe et al. 2011). In addition, several space missions (see Table 1) carried dedicated instruments to map the spatial and size distributions, and in some cases even the composition of interplanetary dust particles throughout the solar system.

Figure 1 shows the mass influx of cosmic solid material entering the Earth's atmosphere and, by extension bombarding the lunar surface and the surfaces of all asteroids near 1 $\mathrm{AU}$, as a function of mass. Labels in Fig. 1 indicate typical methods detecting interplanetary dust. Undoubtedly, our most detailed knowledge on the origin and morphology of the 
Fig. 1 Mass influx (per decade of mass) plotted against particle mass and the observational methods of interplanetary dust (Flynn et al. 2006; Plane 2012)

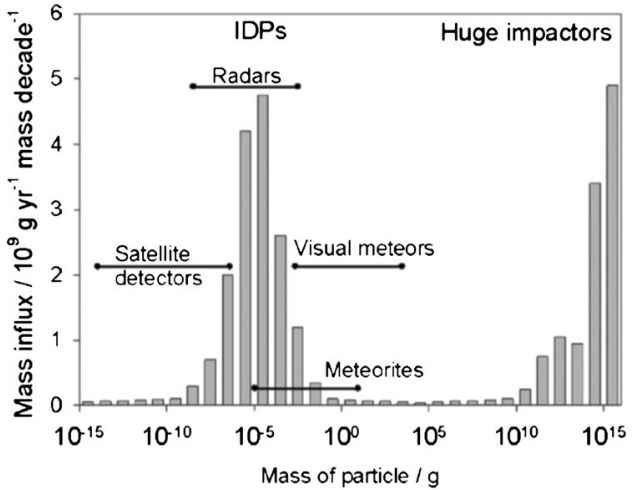

near Earth meteoroid environment comes from ground-based observations, specifically using radars and optical measuring techniques (Janches et al. 2017; Pokorný and Brown 2016; Campbell-Brown and Wiegert 2009; Janches et al. 2015; Campbell-Brown 2015; Baggaley 2002; Kero et al. 2019; Koten et al. 2019; Bruzzone et al. 2020). Most of the ZDC particles form the so-called sporadic meteoroid complex (SMC), and unlike meteor showers, their orbits have evolved significantly over time. This evolution is the product of effects such as Poynting- Robertson (PR) drag, radiation pressure, sublimation, mutual collisions, and the dynamical effects of the planets (Nesvorný et al. 2010; Pokorný et al. 2014). Therefore sporadic meteoroids exhibit a broad distribution of physical properties, in particular those of mass, speed, and orbital parameters. Consequently, it is very difficult to associate sporadic meteors with any particular parent body, unlike the case for many meteor showers (Jenniskens 2006; Janches et al. 2020b; Hajduková and Neslusan 2020), and thus they can only be characterized through statistical measurements of the SMC's interaction with the Earth.

The SMC is characterized by six apparent sporadic meteoroid sources, which correspond to different orbital families of interplanetary dust as seen from an Earth-based observing system (Brown and Jones 1995). These are known as: 1) the North and South Apex (NA \& SA) sources, composed mainly of dust from Halley Type and Oort Cloud Comets (HTCs \& OCCs; Nesvorný et al. 2011b; Pokorný et al. 2014; Sekanina 1976); 2) the Helion (H) and Anti-Helion (AH) sources, composed of dust from Jupiter Family Comets and Main Belt Asteroids (JFCs \& MBAs; Hawkins 1956; Nesvorný et al. 2010, 2011a; Weiss and Smith 1960); and 3) the North and South Toroidal (NT \& ST) sources, composed of dust from HTCs (Campbell-Brown and Wiegert 2009; Jones and Brown 1993; Pokorný et al. 2014; Janches et al. 2015). Other meteoroid populations such as those produced by EdgeworthKuiper Belt objects (EKB) have been shown to be of little significance to the overall flux in the inner Solar System (Poppe 2016).

Figure 2 displays the position of these sporadic meteor radiant distributions as white ellipses on a map in ecliptic coordinates in which the sources are viewed from an Earthcentered frame of reference. Therefore, the radiants are expressed as $\lambda-\lambda_{0}$, where $\lambda$ is the heliocentric ecliptic longitude and $\lambda_{0}$ is the true longitude of the Sun, and $\beta$, the heliocentric ecliptic latitude. This effectively removes the motion of the Earth relative to the Sun, allowing to display the position of each source fixed in heliocentric ecliptic coordinates throughout the year (e.g. the Earth's apex is always at $270^{\circ}$ ). The colored image on the map represents a combined composite year of observations from the two main radars which currently provide continuous surveillance of the near Earth meteoroid environment. These are the Canadian Meteor Orbital Radar (CMOR; Brown et al. 2008) and the Southern Argentina 




Fig. 2 The position of the sporadic meteor radiant distributions in a map of ecliptic coordinates in which the sources are viewed from an Earth-centered frame of reference. Note that the symmetry in intensity in this figure is artificial as the radars are not cross calibrated. To produce this figure the data were normalized such that SAAMER counts matched CMOR for the Helion/Anti-Helion sources and for the SDA meteor shower. The color bar represents the number of meteors within a box of $0.25 \times 0.25$ degree (in $\lambda-\lambda_{0}$ and $\beta$ ). From Janches et al. (2020)

Agile MEteor Radar - Orbital System (SAAMER-OS; Janches et al. 2015; Bruzzone et al. 2020). Note that the radars are not calibrated with each other and thus the intensity of the sources on the colored image are not absolute. The red circles on the figure identify three meteor showers which, although active only for a limited period of time with respect to the SMC, have activity that is well above the SMC background, even when a full year of observations is combined. These are the $\eta$ Aquarids (ETA), the Southern $\delta$ Aquariids (SDA) and the Geminids (GEM). As can be seen, meteor showers are not only constrained in time but also their radiant distributions are more localized in space, while the sporadic sources result in wider distribution present through out the year.

Because these sources will manifest similarly at other inner Solar System bodies (Pokorný et al. 2018; Janches et al. 2018; Pokorný et al. 2019), it is evident from Fig. 2 that the meteoroid influx on planetary bodies is not isotropic. The anisotropic distribution of meteoroids in arrival direction may produce seasonal, diurnal and planetographic variability of incoming meteoroids (Fentzke and Janches 2008; Janches et al. 2018; Pokorný et al. 2017b; Janches et al. 2020). For a limited number of planetary bodies in the solar system it has indeed been demonstrated that this variability, often referred as Meteoroid Input Function (MIF; Fentzke and Janches 2008; Janches et al. 2020), manifests into the directionality of arrival of meteoroids, providing a specific local time and planetographic dependence (Janches et al. 2006), which measurably influences the composition of planetary atmospheres and space weathering of airless bodies (Marsh et al. 2013; Pokorný et al. 2018; Janches et al. 2018; Pokorný et al. 2019). These studies showed the meteoroid changes from body to body and the relative ratio of SMC populations to strongly depend on the orbital and rotational characteristic of the solar system bodies in question.

A great amount of past effort has been expended to model satellite observations of scattered light and thermal infrared emissions from the ZDC and a detailed list of those results are given by Nesvorný et al. (2010). Until recently, most of the models that described the morphology of the sporadic sources were phenomenological in nature and were driven mostly by specific observations (Grün et al. 1985; Dikarev et al. 2004; Fentzke and Janches 2008; Schult et al. 2017; Moorhead et al. 2020). However, dynamical models are better suited to generalize modeling results at different locations in the solar system, since they take into account the physical properties of interplanetary dust to determine, in principle, 
the behavior of particles in interplanetary space independently of any observing methodology (Nesvorný et al. 2010). All these models reproduce the directionality of particles, including the spatial, size and velocity distributions required to replicate observations such as those shown in Fig. 2. This level of description is essential for extrapolating the temporal and planetographic variability of the meteoroid influx on planetary surfaces and atmospheres from present measurements, and for assessing collisional risk with satellites (Fentzke and Janches 2008; Janches et al. 2018; Marsh et al. 2013; Thorpe et al. 2019; Moorhead et al. 2020; Pokorný et al. 2020). Predictions from these models can be constrained using a broad spectrum of methodologies such as direct observations of meteors (Pokorný et al. 2014; Janches et al. 2017; Swarnalingam et al. 2019), observations of atmospheric effects related to the meteor influx (on Earth and other planets; Carrillo-Sánchez et al. 2020), results from micrometeorite collection in Antarctica (Carrillo-Sánchez et al. 2016), surface and vaporization effects produced by impact debris (Cintala 1992; Borin et al. 2009, 2016a,b; Pokorný et al. 2018, 2019) and even satellite anomalies (Thorpe et al. 2019).

In particular, in the past decade a set of dynamical models have reproduced well the main characteristics of the four main inner solar system meteoroid sources. These include the model reported by Nesvorný et al. (2010, 2011a) for particles released by JFCs and MBAs, the model reported by Pokorný et al. (2014) for particles released from HTCs, and the model reported by Nesvorný et al. (2011b) for OCCs released meteoroid. Similar dynamical models have been reported by Wiegert et al. (2009) and Poppe (2016). The first one considers a limited number of JFCs to mainly understand CMOR observations, while the latter includes the four population as well as EKB objects and it is mainly constrained with limited statistics from dust detector measurements on board satellites in the outer Solar System.

Dynamical models track the temporal and orbital evolution of particles ejected from sources (JFCs, MBA, HTCs, and OCCs) to sinks (sublimation, impact in a solar system body, collisional destruction, or ejection from the solar system body). They include both gravitational perturbations by planets and relevant non-gravitational effects, such as radiation pressure and PR drag. They also incorporate particle collisions following treatments such as those proposed by Steel and Elford (1986) and/or collisional grooming (Stark and Kuchner 2009). Cloud density is constrained with a size distribution function (SDF) determined with, for example, LDEF (Love and Brownlee 1993) observations assuming a logarithmic shape (at $1 \mathrm{AU}$ well supported by radar observations of meteors, see Janches et al. 2019, and references therein). Some of the models treat the collisional lifetimes and SDF at the source as adjustable parameters, and methods like that proposed by Kessler (1981) are used to calculate the impact probability with planetary bodies. Overall, these models have concluded that a majority ( $\sim 85-95 \%)$ of the ZDC near 1 AU originates from JFCs (e.g., Levison and Duncan 1997), while MBA, HTC, and OCC meteoroids contribute the remaining 5-15\%. Initial constrains using ZDC infrared emissions detected by the InfraRed Astronomical Satellite (IRAS; Nesvorný et al. 2011a); lidar measurements of the global input of neutral Na and Fe measured at Earth (Gardner et al. 2014; Carrillo-Sánchez et al. 2016); and the flux derived from cosmic spherules collected in Antarctica (Taylor et al. 1998) support these contributions, but they are still challenged by other measurements (see Introduction in Janches et al. 2014). Thus using meteoroid related phenomena at airless bodies like Mercury and the Moon, such as dust clouds and exospheric emissions, offer a unique way to further constrain these critical quantities.

Finally, in addition to the parameters mentioned above - number flux, radiant and mass distributions -, the impact velocity of some meteoroid populations may play a significant role in the formation of exospheres. Figure 3 shows modeled velocity distributions at $1 \mathrm{AU}$ for the four dominant meteoroids populations in the inner solar system. In particular, the 

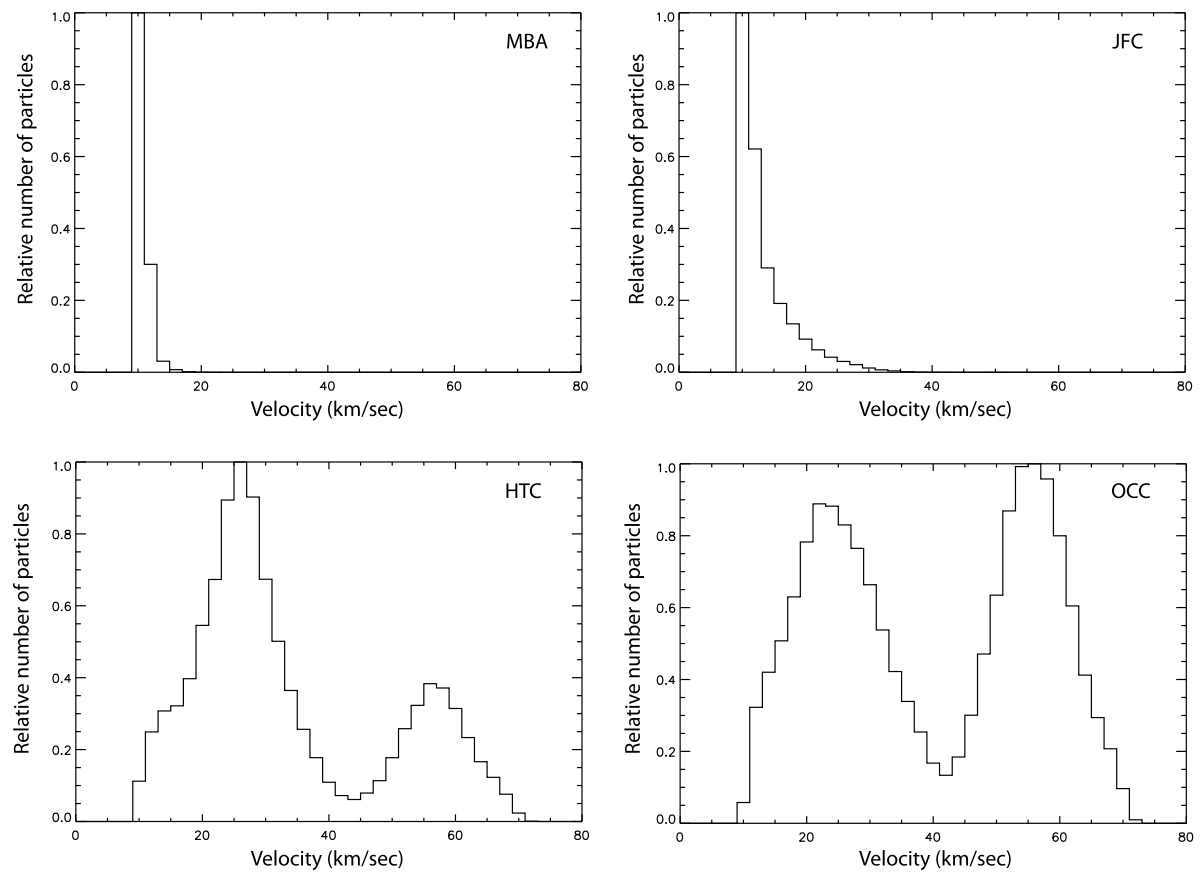

Fig. 3 Velocity distributions at $1 \mathrm{AU}$ from the four main populations of meteoroids

lower velocity peak $\left(\sim 25 \mathrm{~km} \mathrm{~s}^{-1}\right)$ of the bi-modal distributions of the long period comets populations (i.e., HTCs and OCCs) corresponds to meteoroids in the toroidal region, while the faster distribution $\left(\sim 55 \mathrm{~km} \mathrm{~s}^{-1}\right)$ corresponds to retrograde orbits in the Earth's apex direction. As can be seen in the panels of Fig. 3, these distributions are significantly different at $1 \mathrm{AU}$ and, as it will be shown in the next sections, the physical and orbital differences of Mercury and the Moon are even more extreme.

\section{Meteoroid Influx at Mercury}

Meteoroid impact vaporization has long been considered to be an important source of metals in Mercury's exosphere. Metal constituents of this exosphere have been observed using ground-based and spaceborne techniques. A detailed summary of observations is presented in Bida and Killen (2017) and Johnson and Hauck (2016). The importance of impact vaporization became clearer when measurements by the UltraViolet and Visible Spectrometer (UVVS) instrument on board the NASA MESSENGER spacecraft permitted the study of the seasonal variations of refractory metals like Mg and Ca (Vervack et al. 2010; Sarantos et al. 2011; Burger et al. 2014; Merkel et al. 2017). Detections of metal emissions of $\mathrm{Al}$ and Fe have also been recently reported (Vervack et al. 2016; Bida and Killen 2017) utilizing ground-based telescope and MESSENGER data. Although the relative importance of sources for Mercury's exosphere is detailed in companion papers to this, from data and models, three lines of evidence point to impact vaporization as an important source of these atoms. First, altitude profiles of neutral densities yield source temperatures that appear consistent with meteoroid impacts. Low temperature components are consistent with vaporiza- 
tion of atoms from Mercury's soil, and high temperatures are consistent with molecules that quickly dissociate. Second, the dependence of the inferred source rate for $\mathrm{Ca}$ and $\mathrm{Mg}$ with Mercury's True Anomaly Angle (TAA) approximately matches predictions of how the impact vapor varies with heliocentric distance (Killen and Hahn 2015; Pokorný et al. 2018). And third, the morphology of $\mathrm{Mg}$ and $\mathrm{Ca}$ emissions appears to be consistent with impact vaporization because both $\mathrm{Ca}$ and $\mathrm{Mg}$ exhibit a pronounced dawn-dusk asymmetry (McClintock et al. 2009; Burger et al. 2014; Merkel et al. 2017).

Numerous reports have utilized the numerical modeling of both meteoroid mass and flux distributions to explain their effects on airless bodies, especially at Mercury and the Moon. The main difference between these works is what populations they include. As discussed in Sect. 2, this effectively changes two critical factors to understand exospheric formation: 1) the impact velocity; and 2) the directionality of incoming flux. The first factor was identified by Cintala (1992), while the second one was shown to be crucial at the Moon by LADEE observations (Szalay and Horányi 2015). As more comprehensive data becomes available (e.g., LDEX) more details of these complex interactions is revealed, enabling to discern more accurately the role that each population play in producing the observables.

Asteroidal meteoroids are perhaps the population that has been more widely studied as one of the sources of the hermean exosphere, starting from the work reported by Cintala (1992) and later by Marchi et al. (2005). This is because it was believed that MBA meteoroids dominated the flux at Earth, in comparison to particles from cometary sources (Ceplecha 1992). However, this has been challenged recently by Nesvorný et al. (2010, 2011a) who argued that meteoroids originating from short period comets (i.e., JFCs) dominate the inner solar system in mass flux, number flux, and total cross section in the micrometer to millimeter range. Although this matter is far from settled, it certainly emphasizes the need for considering all populations.

Cintala (1992) considered particles with sizes smaller than $1 \mathrm{~cm}$ and a mean impact velocity on Mercury of about $20 \mathrm{~km} \mathrm{~s}^{-1}$ (Fig. 4), while Marchi et al. (2005) treated larger MBA meteoroids $(D>2 \mathrm{~cm})$ which arrive with a much broader impact velocity distribution at Mercury, ranging from 4.25 to around $40 \mathrm{~km} \mathrm{~s}^{-1}$ (Fig. 4). The higher velocities are due to the fact that larger particles are only influenced by gravitational forces exerted by mean motion and secular resonances and, thus, in order to reach small heliocentric distances they would need to be ejected on highly eccentric orbits similar to near-Earth asteroids with low perihelia (Pokorný et al. 2018). Nevertheless, the larger particles are likely of little significance as the main source of the permanent exosphere because they are infrequent while the exosphere must be continuously replenished. Small particles originating from MBAs ( $D<2 \mathrm{~cm}$ ) were re-visited by Borin et al. $(2009,2016 \mathrm{a}, \mathrm{b})$ who showed that the circularization of their orbits by PR drag result in narrow impact velocity distributions (Fig. 4). The analytic velocity distribution of Cintala (1992) is $20.50 \mathrm{~km} \mathrm{~s}^{-1}$ as compared to $16.81 \mathrm{~km} \mathrm{~s}^{-1}$ from Borin et al. (2009, 2016a,b).

More recently, Pokorný et al. (2017b) demonstrated for the first time how the directionality of the meteoroid influx relates to the characteristics of the hermean exosphere. This work pointed out the strong dependence of impact characteristics and fluxes with respect to the planet's TAA. Although such dependence is present in all planets and satellites (Janches et al. 2018, 2020), it is particularly evident at Mercury due to the planet's high eccentricity and orbital inclination (Pokorný et al. 2017b). Furthermore, Pokorný et al. (2018) utilizing the description of MBA-released meteoroids reported by Nesvorný et al. (2010), showed the strong effect that such dependency has on impact velocities. The authors estimated that these particles, as well as JFC-released meteoroids, impact the hermean surface preferentially with lower eccentricities $\mathrm{e}<0.2$ and small inclinations $\mathrm{I}<30^{\circ}$, and much larger impact 
Fig. 4 Velocity distribution function of particles, produced in the Main Belt, with radius of $5 \mu \mathrm{m}$ and $100 \mu \mathrm{m}$ (Borin et al. 2009)

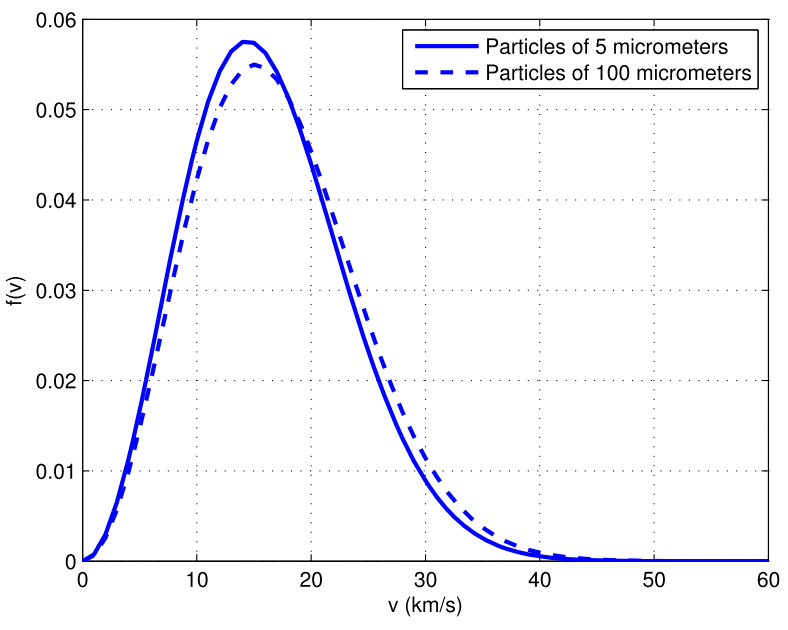

velocities than those estimated by Cintala (1992) and Borin et al. (2009, 2016a,b). Specifically, Pokorný et al. (2018) estimated MBA and JFC meteoroids having impact velocities as large as $\mathrm{V}_{\text {imp }}<70 \mathrm{~km} \mathrm{~s}^{-1}$ at perihelion and $\mathrm{V}_{\text {imp }}<50 \mathrm{~km} \mathrm{~s}^{-1}$ at aphelion.

Regarding JFC released meteoroids, Nesvorný et al. (2010) emphasized the importance of this source in the inner solar system. Both, Borin et al. (2016a,b, 2017) and Pokorný et al. (2017b, 2018) considered for the first time this population as part of the influx at Mercury. Due to the high eccentricity of Mercury and low impact velocities of these meteoroids compared to Mercury's orbital velocity, the impact directions of JFC and MBA meteoroids are expected to experience significant motion in the local time reference frame during Mercury's orbit. This is shown in Fig. 5, where the radiant distributions for JFC meteoroids with diameter $\mathrm{D}=10 \mu \mathrm{m}$ are displayed for six TAAs. It can be seen that at perihelion the meteoroid flux is concentrated around $\left(6 \mathrm{hr}, 60^{\circ}\right)$ as a result of the nonzero inclination of Mercury's orbit and the orientation of the Hermean velocity vector. Similarly to MBA meteoroids (see Pokorný et al. 2018), there is a shift in the radiant distribution of JFC meteoroids as Mercury moves toward or away from the Sun, caused by the nonzero eccentricity and inclination of Mercury's orbit and a consequent drift of the planet's velocity vector from the ecliptic plane and its perpendicular orientation with regard to the radial vector.

Regarding meteoroids from long period comets (LPC; i.e., HTC and OCC), Pokorný et al. (2017b, 2018) showed for the first time their essential role in the formation of Mercury's exosphere. Dynamically less evolved than MBA or JFC meteoroids and released into highly eccentric orbits, LPC meteoroids can reach orbits intersecting Mercury faster and at high speeds. In fact, Pokorný et al. (2018) showed that these populations, like at Earth, encounter Mercury with a flat eccentricity distribution and a bi-modal distribution of orbital inclinations of prograde and retrograde orbits. In particular, the retrograde portion of the HTC and OCC populations impact Mercury's surface from the apex direction, with velocities as high as $95 \mathrm{~km} \mathrm{~s}^{-1}<\mathrm{V}_{\text {imp }}<120 \mathrm{~km} \mathrm{~s}^{-1}$ at perihelion and $75 \mathrm{~km} \mathrm{~s}^{-1}<\mathrm{V}_{\text {imp }}<90 \mathrm{~km}$ ${ }^{-1}$ at aphelion, and are less influenced by Mercury's orbital motion. The high impact velocity of these particles makes them critical for the morphology of the exosphere at Mercury and, in fact, Pokorný et al. (2017b, 2018) demonstrated a persistent dawn enhancement of the dust/meteoroid environment at Mercury, which should be responsible of the dawn-dusk asymmetry in Mercury's Ca exosphere (Fig. 6). 

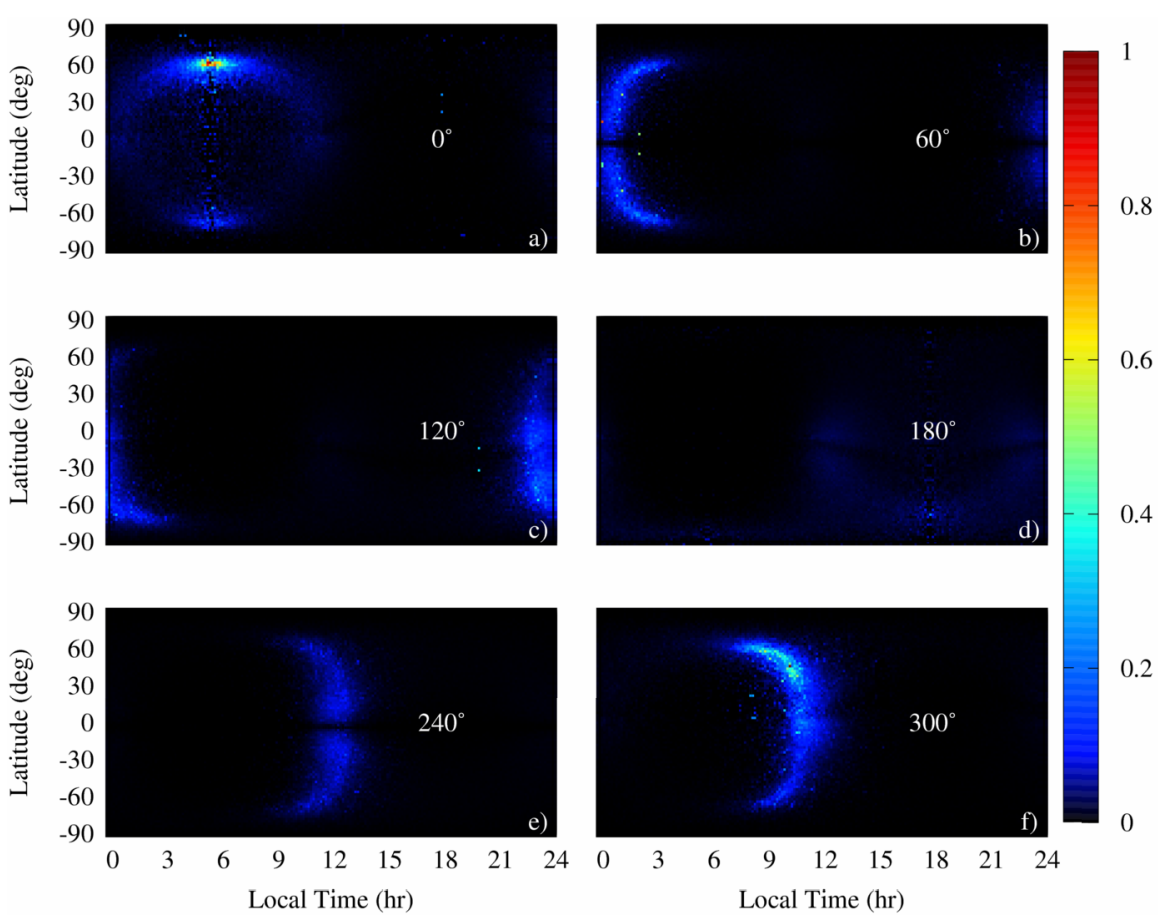

Fig. 5 Normalized radiant distribution of $D=10 \mu \mathrm{m}$ JFC meteoroids impacting Mercury's surface for six different TAAs (white number indicated in each panel). The mutual meteoroid collisions are not considered in this case. The $\mathrm{x}$-axis represents the local time on Mercury, and it is fixed with regard to the subsolar point (12 hr). Due to Mercury's eccentricity, the location of the apex (approximately at $6 \mathrm{hr}$ ) changes along Mercury's orbit. The latitude is measured from Mercury's orbital plane (not the ecliptic). From Pokorný et al. (2018)

The high impact velocity at Mercury of LPC meteoroids, resulting from retrograde orbits (Levison et al. 2006), makes them the dominant source of physical phenomena regarding the formation and morphology of its exosphere. This is interesting because at the same time they are not considered to be a dominant part of the inner solar system meteoroid budget in terms of the mass flux, number flux, or total meteoroid cross section (Nesvorný et al. 2011b; Pokorný et al. 2014; Carrillo-Sánchez et al. 2016). Specifically, the mass flux of LPC meteoroids at Mercury compared to JFC meteoroids could be as small as 5\% but their impact velocities resulting in values over $100 \mathrm{~km} \mathrm{~s}^{-1}$ makes them the dominant source in terms of the impact vaporization or the impact yield (Pokorný et al. 2017b, 2018).

Besides the orbital and physical characteristics of the meteoroid influx on Mercury and other bodies, a last crucial quantity to consider is the total meteoric mass impinging on the planet, and observations can help constrain better that hotly debated quantity (see Table 1 in Plane 2012). It is important to note that absolute fluxes are heavily dependent on the measurements used to constrain those values (Nesvorný et al. 2010; Carrillo-Sánchez et al. 2016; Janches et al. 2017; Carrillo-Sánchez et al. 2020). In addition, the agreement found with those measurement constraints is highly dependent on the uncertainties of the model parameters such as the assumed collisional lifetimes, particle densities and/or SDF at the source, which can be large (Pokorný et al. 2018, 2019). Naturally, the most convenient 


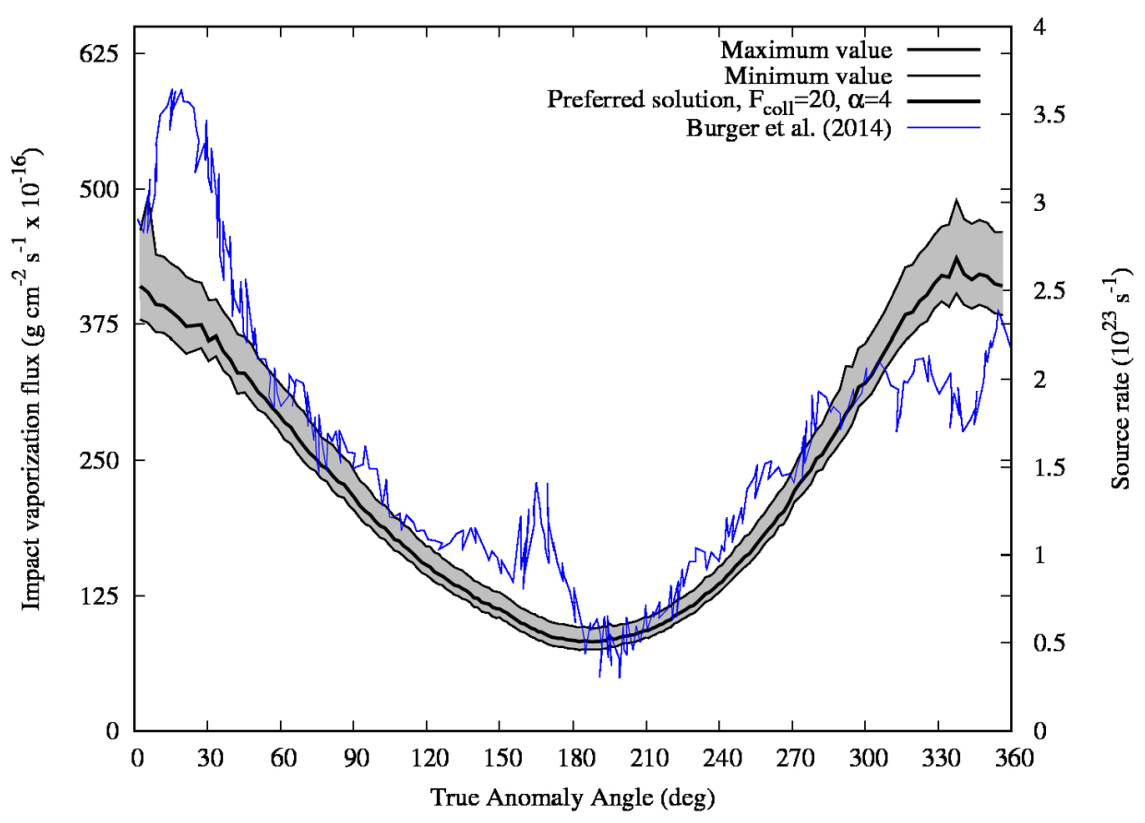

Fig. 6 Seasonal variations of the relative vaporization rate from a dynamical model (solid thick black line is the preferred solution and the confidence interval is marked by the gray area and thin black lines) compared to measurements of exospheric abundance of Ca from Burger et al. (2014) (solid blue lines). From Pokorný et al. (2018)

pivot/anchor point (i.e. something that everything else is relative to) is usually the Earth (and the mass flux on Earth), due to the significant wealth of data for the Earth/Moon region.

Cremonese et al. (2012), for example, considered the measurements of the dimensions of all the hypervelocity impact craters collected on the space-facing end of the gravitygradient-stabilized LDEF satellite (Love and Brownlee 1993; Miao and Stark 2001), in order to determine the mass flux of extraterrestrial micrometeoroids at Earth in the submillimeter diameter range of 10-500 $\mu \mathrm{m}$. The first step for translating the crater data on LDEF into a flux estimate is to interpret the crater diameters in terms of projectile size. Love and Brownlee (1993) have calculated the meteoroid mass distribution using a polynomial fit to the crater size-frequency distribution along with the mean depth-diameter ratio as well as a single mean meteoroid impact velocity and angle. It is important to point out that the velocity and impact angle are initially unknown parameters and, therefore, need to be fixed to infer the mass distribution from the crater sizes. In fact, Christiansen (1992) reported laboratory impact experiments of projectiles striking thick aluminum alloy targets at speeds up to $18 \mathrm{~km} \mathrm{~s}^{-1}$, demonstrating that crater volume under those conditions is nearly proportional to the projectile kinetic energy. Love and Brownlee (1993) chose the average meteoroid speed to be $16.9 \mathrm{~km} \mathrm{~s}^{-1}$, as found by Erickson (1969) and Kessler (1969) from photographic meteors and supported by crater rate measurements on LDEF. It is important to note that, the latter two assumptions have been revised by several authors (Taylor 1995; Mathews et al. 2001; Miao and Stark 2001). Cremonese et al. (2012) adopted a semiempirical scaling law derived from laboratory experiments that were performed in a range of velocities that were significantly different with respect to the impact speeds of the impacting meteoroids. To improve the analysis of LDEF data the relation between crater diameter and projectile size has 
been specifically explored with the hydrocode iSALE. The ratio between the depth and diameter of the craters is 0.527, accurately measured by Love and Brownlee (1993) on LDEF, and it has been considered as an important constraint for the iSALE simulations (Cremonese et al. 2012). The resulting average ratio derived by iSALE simulations between depth and diameter is 0.582 for asteroids. This value depends on the precision of the hydrocode simulations, which according to code validation against laboratory experiments (Pierazzo et al. 2008 ), is $3-4 \%$ in radius and $12 \%$ in depth.

Using the dynamical model of dust particle orbital evolution described in Borin et al. (2009), the range of impact velocities used in the hydrocode simulations were derived from which Cremonese et al. (2012) calculated the total mass accreted by the Earth of (11.5 \pm 1.4$)$ $t . d^{-1}$ assuming cometary dust and $(20.3 \pm 2.7) t . d^{-1}$ assuming asteroidal dust, where $t$ represents a metric ton $(1000 \mathrm{~kg})$. Borin et al. (2009) extrapolated the curve of the Earth meteoroid flux, obtained by Cremonese et al. (2012), to Mercury assuming the impact velocity distribution shown in Fig. 4. The derived asteroidal mean flux value is $8.20 \pm 0.75 \times 10^{-15}$ $\mathrm{g} \mathrm{cm}^{-2} \mathrm{~s}^{-1}$ (Borin et al. 2017).

Pokorný et al. (2018) followed a somewhat different approach by treating the four main populations of meteoroids separately. These authors estimated that Mercury accumulates $7 \%$ of MBA meteoroid mass as compared to Earth due to their low-eccentricity orbits, with low relative impact velocities of this population onto both planets. Such low velocities are efficiently attracted by Earth's gravity, while this effect is much smaller for Mercury. In addition, JFC meteoroids, on the other hand, have a broader distribution of eccentricities, which weakens the gravitational focusing and leads to higher mass accretion $(\sim 23 \%)$ at Mercury compared to that at Earth. The meteoroids produced by HTCs and OCCs, on the other hand, have significantly higher Mercury-to-Earth mass accretion ratios as compared to JFC meteoroids, $70 \%$ for HTC meteoroids and $\sim 90 \%$ for OCC meteoroids. In particular, Pokorný et al. $(2018,2019)$ explored the effects on the production of the ejecta dust cloud on Mercury and the Moon, respectively, produced by different Size Distribution Functions (SDF) resulting from varying the differential size index, $\alpha$ (i.e. exponent of the SDF) and different collisional lifetimes resulting from using a collisional fudge factor, $F_{\text {coll }}$. The authors defined a preferred solution adopting $\alpha=4$ (or a differential mass index, $s=(\alpha+2) / 3$ $=2$ in agreement with radar observations; Janches et al. 2019) and $F_{\text {coll }}=20$, shown to provide the best agreement with measurements (Pokorný et al. 2019). This solution also used the contribution ratios of the different populations at Earth determined by Carrillo-Sánchez et al. (2016) which were estimated subject to the following assumptions: 1) According to lidar measurements, the global input of neutral $\mathrm{Na}$ and Fe measured at Earth (Gardner et al. 2014) is estimated to be $0.3 \pm 0.1 t . d^{-1}$ and $2.3 \pm 1.1 t . d^{-1}$, respectively; and 2) The flux of cosmic spherules with diameters between $50 \mu \mathrm{m}$ and $700 \mu \mathrm{m}$ is estimated to be $4.4 \pm 0.8$ $t . d^{-1}$ (Taylor et al. 1998). Consequently, Carrillo-Sánchez et al. (2020) determined a total mass input of $27.9 \pm 8.1 t . d^{-1}$ for Earth, with JFC meteoroids being the main contributor to this flux $(\sim 70 \%)$.

Based on these estimates and assumptions, Pokorný et al. (2018) provided the following values of accreted mass averaged over the entire Hermean orbit: MBA meteoroids $M_{M B A}=0.26 \pm 0.15 t . d^{-1}$, JFC meteoroids $M_{J F C}=7.84 \pm 3.13 t . d^{-1}$, HTCs $M_{H T C}=1.69 \pm 0.91$ $t . d^{-1}$ and OCCs $M_{O C C}=2.37 \pm 1.38 t . d^{-1}$. This represents a mass influx ratio of JFC/LPC meteoroids of $\sim 2$, which is much lower than that at Earth $(\sim 7)$ according to CarrilloSánchez et al. (2016, 2020).

One constraint for these estimated values at Mercury is the impact vaporization flux. The vaporization flux averaged over a hermean year results in $F_{\text {orbit }}=(200 \pm 16) \times$ $10^{-16} \mathrm{~g} \mathrm{~cm}^{-2} \mathrm{~s}^{-1}$, with maximum value of $(436 \pm 57) \times 10^{-16} \mathrm{~g} \mathrm{~cm}^{-2} \mathrm{~s}^{-1}$ occurring at 
$\mathrm{TAA}=337^{\circ}$, and a minimum value of $(82 \pm 12) \times 10^{-16} \mathrm{~g} \mathrm{~cm}^{-2} \mathrm{~s}^{-1}$ occurring at TAA $=188^{\circ}$. Although, these values represent impact vaporization fluxes of the same order of magnitude as those reported by Borin et al. (2009, 2017), the similarity is misleading because Pokorný et al. (2018) predicted a lower mass flux of meteoroids at Mercury with respect to those modeled by Borin et al. (2009, 2017) (Mercury/Earth ratio less than 1 vs. 35) accompanied by higher-speed distributions. The variations of the impact vaporization flux and the impact directionality also contradict the result of Borin et al. $(2009,2017)$ because the model reported by Pokorný et al. (2018) predicts a larger perihelion-to-aphelion ratio in the impact vaporization rate (Fig. 7). Regardless of these details, all estimates of impact vaporization rates more than suffice to supply Mercury's exosphere: compared to source rates derived for $\mathrm{Ca}$ and Mg from MESSENGER measurements (Sarantos et al. 2011; Burger et al. 2014; Merkel et al. 2017), only $\sim 1 \%$ of the estimated total vapor appears to contribute neutrals to Mercury's exosphere.

While future measurements by the Bepi Colombo spacecraft will further constrain our models of the meteoroid environment at Mercury, there are currently two constraints to assess the validity of our estimates of total exospheric context with Mercury's heliocentric distance. Pokorný et al. (2018) showed a strong dawn/dusk asymmetry in both meteoroid impact direction distribution and the impact vaporization pattern on the surface. According to that work, the impact vaporization pattern is expected to undergo some motion during Mercury's orbit, mainly reflecting the precipitation pattern of LPC particles because of their high impact speeds. The total impact vaporization flux integrated over the whole surface follows a similar pattern with TAA to source rates for calcium presented by Burger et al. (2014), with a few enhancements, probably due to meteor shower activities, that will be discussed in Sect. 6.2. As a final note, it is important to note that Ca column density in the exosphere of Mercury obtained by Burger et al. (2014) is not linearly proportional to meteoroid mass flux. The reasons for the deviation of this dependence from a linear function is that $\mathrm{Ca}$ delivery rate to the exosphere is a complex function of quenching temperature of condensation of Ca-containing species, photolysis lifetimes of $\mathrm{CaO}, \mathrm{CaOH}, \mathrm{Ca}(\mathrm{OH})_{2}$ as well as initial temperature and pressure in the impact-produced hot cloud, target-to-impactor mass ratio, typical mass of impacted meteoroids, and the elemental composition of the surface of Mercury and impactors (Berezhnoy 2018).

\section{Meteoroid Influx at the Moon}

Meteoroids directly reach the surface of airless bodies, becoming impact debris, generating clouds of secondary ejecta particles, and leaving a crater record on the surface. These phenomena provide data to indirectly evaluate the links between measured effects on the lunar surface and meteoroid influx. For example, Grün et al. (1985) used these craters to decipher the meteoroid size distribution impinging on the lunar surface. The majority of the ejecta particles have initial speeds below the escape speed from the Moon $\left(2.4 \mathrm{~km} \mathrm{~s}^{-1}\right)$ and following ballistic orbits return to the surface, blanketing the lunar crust with a highly pulverized regolith with $\gg 1 \mathrm{~m}$ thickness. Micron and sub-micron sized secondary particles that are ejected with speeds up to the escape speed form a highly variable, but permanently present, dust cloud around the Moon. Such tenuous clouds have been observed by the Galileo spacecraft around all lunar-sized Galilean satellites at Jupiter (Krüger et al. 2003). These observations have been the source of progress regarding the lunar dust environment because only limited new observational data at the Earth's natural satellite has been obtained since the Apollo era (Grün et al. 2011). In this manuscript, we will focus on what we have learned 


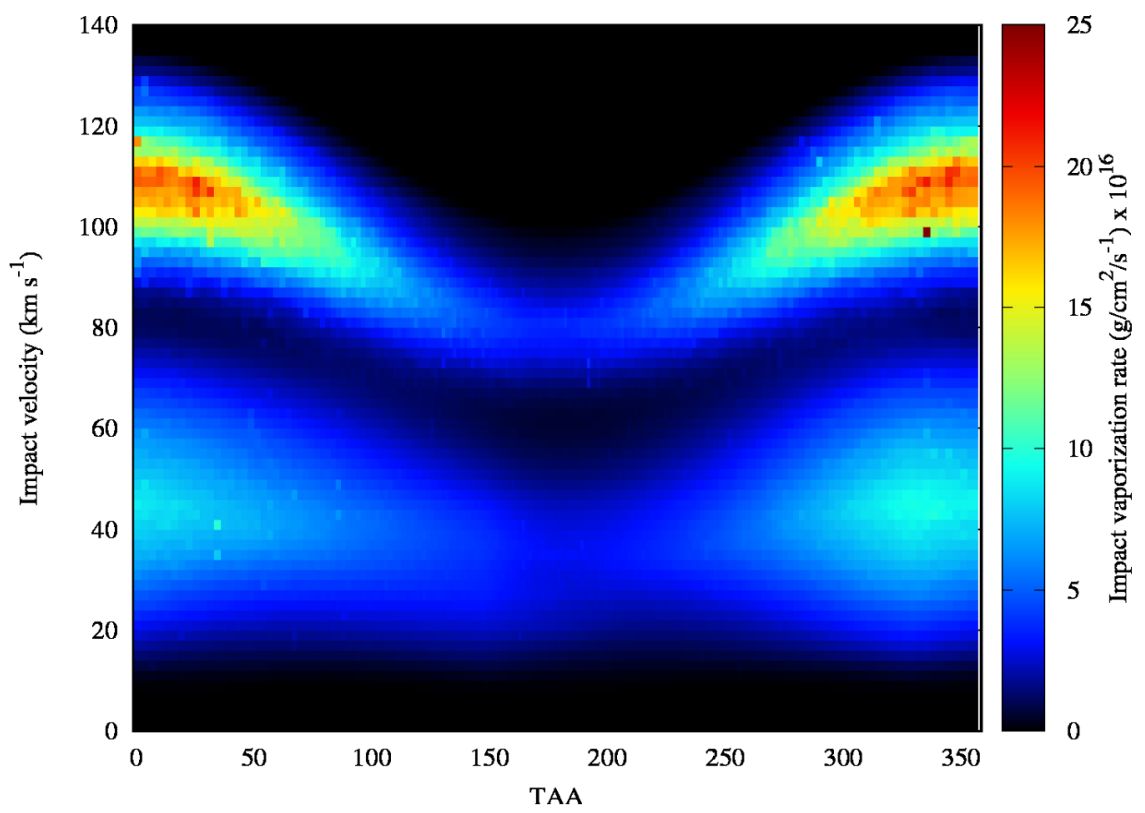

Fig. 7 Total vaporization flux as a function of TAA (x-axis), and the impact velocity (y-axis). The units are $\mathrm{g} \mathrm{cm}^{-2} \mathrm{~s}^{-1}$ per $2 \mathrm{~km} \mathrm{~s}^{-1}$ bin. From Pokorný et al. (2018)

during the era following the LADEE mission, which greatly enhanced our view of the highaltitude $(\gg 1 \mathrm{~km})$ lunar dust environment (Elphic et al. 2014). This mission provided key measurements to better constrain our models of the meteoroid environment at $1 \mathrm{AU}$ as well as to define more accurately the connection between meteoroid bombardment and exosphere formation, thus defining a 'before and after' reference point on our knowledge of the meteoroid environment influence on the lunar surface.

LADEE, launched in September 2013, followed a near-equatorial retrograde orbit, with a characteristic orbital speed of $1.6 \mathrm{~km} \mathrm{~s}^{-1}$. LADEE was designed to make measurements of the dust environment independently from the lunar exosphere and those observations covered a limited latitude range. Even considering these limitations, LADEE is probably the first mission with a synergy in its measuring capabilities such that the connection between meteoroid bombardment and exospheric formation on an airless solar system body could be investigated (see discussion regarding the Geminids meteor shower in Sect. 6.2). LADEE was designed to directly measure the ejecta cloud generated by meteoroid impacts on the lunar surface. This included possible intermittent density enhancements during meteoroid showers, and searching for the putative regions with high densities of dust particles with radii $\ll 1 \mu \mathrm{m}$ lofted above the terminators (Horányi et al. 2015). The Lunar Dust Experiment (LDEX) on board LADEE was an impact ionization dust detector that measured both the positive and negative charges of the plasma cloud generated when a dust particle strikes its target. The amplitude and shape of the waveforms (signal versus time) recorded from each impact were used to estimate the mass of the dust particles. The instrument had a total sensitive area of $0.01 \mathrm{~m}^{2}$, gradually decreasing to zero for particles arriving from outside its field-of-view of $\pm 68^{\circ}$ off from the normal direction (Horányi et al. 2014). LDEX detected a total of approximately 140,000 dust hits (Fig. 8) during about 80 days of cumulative observation time by the end of the mission in April 2014. 


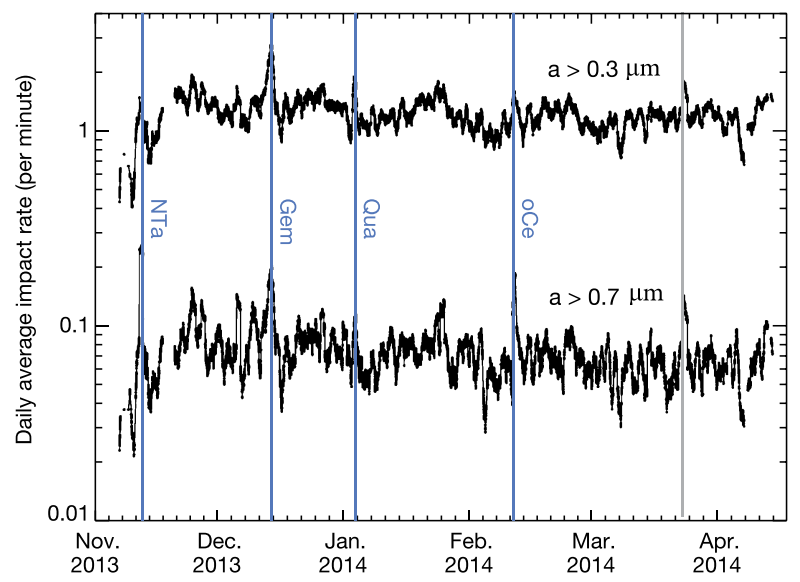

Fig. 8 Impact rates observed by LDEX throughout the duration of the LADEE mission. The panel shows the daily running average of impacts per minute of particles with radii $>0.3 \mu \mathrm{m}$ and $a>0.7 \mu \mathrm{m}$. Four of the annual meteoroid showers generated elevated impact rates that lasted several days. The labelled annual meteor showers (blue vertical lines) are: the Northern Taurids (NTA); the Geminids (GEM); the Quadrantids (QUA); and the Omicron Centaurids (oCe). Towards the end of March LDEX data indicated a meteor shower that remained unidentified (vertical grey line) by ground based observers Horányi et al. (2015)

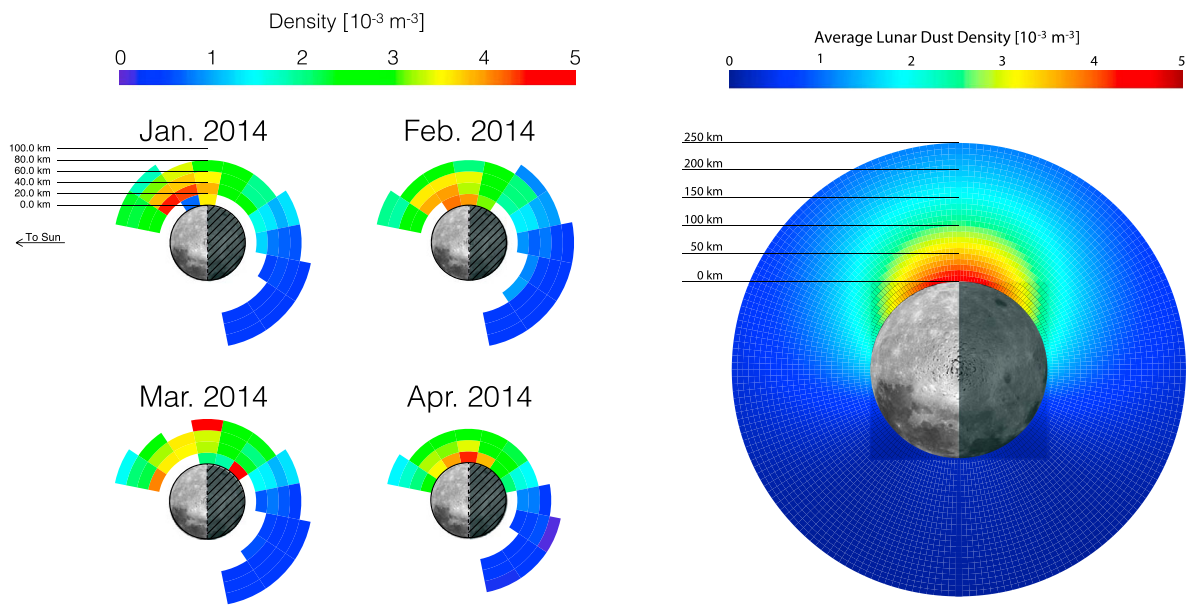

Fig. 9 Left: The average dust ejecta cloud density observed by LDEX for each calendar month for which LADEE was operational in 2014. Each color ring corresponds to the density every $20 \mathrm{~km}$ Szalay and Horányi (2015b). Right: The modeled annually averaged lunar dust density distribution for particles with $a \geq 0.3 \mu \mathrm{m}$. These plots are in a reference frame where the Sun is on the left (-x direction) and the apex motion of the Moon about the Sun is towards the top of the page (+y direction) Szalay and Horányi (2016c)

LDEX measurements provided compelling evidence that our understanding of how meteoroids influence the lunar surface must be revisited. First, the measured fluxes showed that the Moon is engulfed in a permanently present, but highly variable dust exosphere (Fig. 9). Specifically, LDEX data showed that the lunar secondary dust ejecta cloud is persistent and asymmetric, and significantly denser at 5-8 hrs of lunar local time, with a peak density tilted 
somewhat sunward of the dawn terminator. Later, Szalay and Horányi (2015) used an empirical model to show that in order to explain the measurements reported by Horányi et al. (2015), LPC-produced meteoroids (i.e. HTC and OCC) should play a major role in the production of the observed ejecta cloud in the Moon's equatorial plane. The authors argued that the cloud is primarily produced by impacts from a combination of the three known sporadic meteoroid sources (Helion, Anti-Helion, and Apex, See Sect. 2). Furthermore, the cloud density is modulated by both the Moon's orbital motion about the Earth and about the Sun. The tilting of the ejecta cloud toward the Sun was more pronounced earlier in the LADEE mission (November 2013), while the LDEX signal became more centered around the dawn terminator toward the end of the mission (April 2014). From these data features, Szalay and Horányi (2015) inferred a variable relative strength between the Apex, Helion, and AntiHelion sources to account for the change in the structure of the ejecta cloud throughout the mission. The ejecta mass production rate from the Helion source was found to be approximately twice as strong as the Anti-helion source (Szalay and Horányi 2015), which was a puzzling finding because such asymmetry has never been observed in the distribution of meteors measured by Earth-based radars (Campbell-Brown 2008; Janches et al. 2015) and optical systems (Jenniskens et al. 2016).

Efforts of modeling the influence of meteoroids on the lunar surface parallel those at Mercury and differ again on the meteoroid populations included in the different treatments. For example, Cremonese et al. (2013) reported the production of neutral sodium on the Moon caused by impacts of MBA meteoroids with size between 5-100 $\mu \mathrm{m}$ using the same dynamical model of Mercury and reported by Borin et al. (2009) (see Sect. 3). The authors estimated a production rate of $\mathrm{Na}$ atoms of $1.648 \times 10^{5}$ atoms.cm ${ }^{-2} \cdot \mathrm{s}^{-1}$, concluding that the impact process due to meteoroids plays a very important role in the contribution of neutral atoms to the lunar exosphere. Previous reports suggested impact vaporization to be a negligible source to the total column abundance of sodium near noon, with a contribution of $\sim 1 \%$, compared to other processes such as photon-stimulated desorption (PSD; Sarantos et al. 2010), with a measurable fraction $(\sim 50 \%)$ of total column density near the dawn terminator. Similar to the case of Mercury, Borin et al. (2017) updated this model by including cometary particles from short period comets (JFC), but again using a slow velocity distribution (Fig. 4) and lacked sufficient statistics to give distributions of arrival direction.

More recently, Janches et al. (2018) utilized the dynamical models of meteoroids released by JFC, HTC and OCC developed to explain the SMC sources (Nesvorný et al. 2010, 2011a,b; Pokorný et al. 2014) for the purpose of re-interpreting the Szalay and Horányi (2015) results. This effort concluded that $\sim 20 \%$ of the asymmetry present in LDEX measurements is due to unaccounted-for biases introduced by the orientation of the LADEE spacecraft orbit with respect to the selenographic latitudes where the ejecta cloud produced by JFC meteoroids is largest. These modeling results also show that the response of the lunar soil to incoming meteoroids should be necessarilly different on the day and night sides of the Moon, in order to fit correctly the ejecta mass production rates measured by LDEX. The work inferred a smaller mass flux ratio between the short and long-period comet meteoroids on the Moon than that reported at Earth (Moon, 1.3:1 vs. Earth, 7:1). This finding was interpreted to indicate that the ejecta rate yield is a steeper function of the velocity of the incident meteoroids than assumed before, since LPC particles have on average higher impact velocities than their short-period comet counterparts.

Later, Pokorný et al. (2019) expanded on this effort and probed the effects of various free parameters intrinsic to the dynamical models used in Janches et al. (2018) including the effect of gravitational focusing that plays a significant role in shaping the lunar and terrestrial meteoroid environment. Although the model was able to reproduce night-side observations 

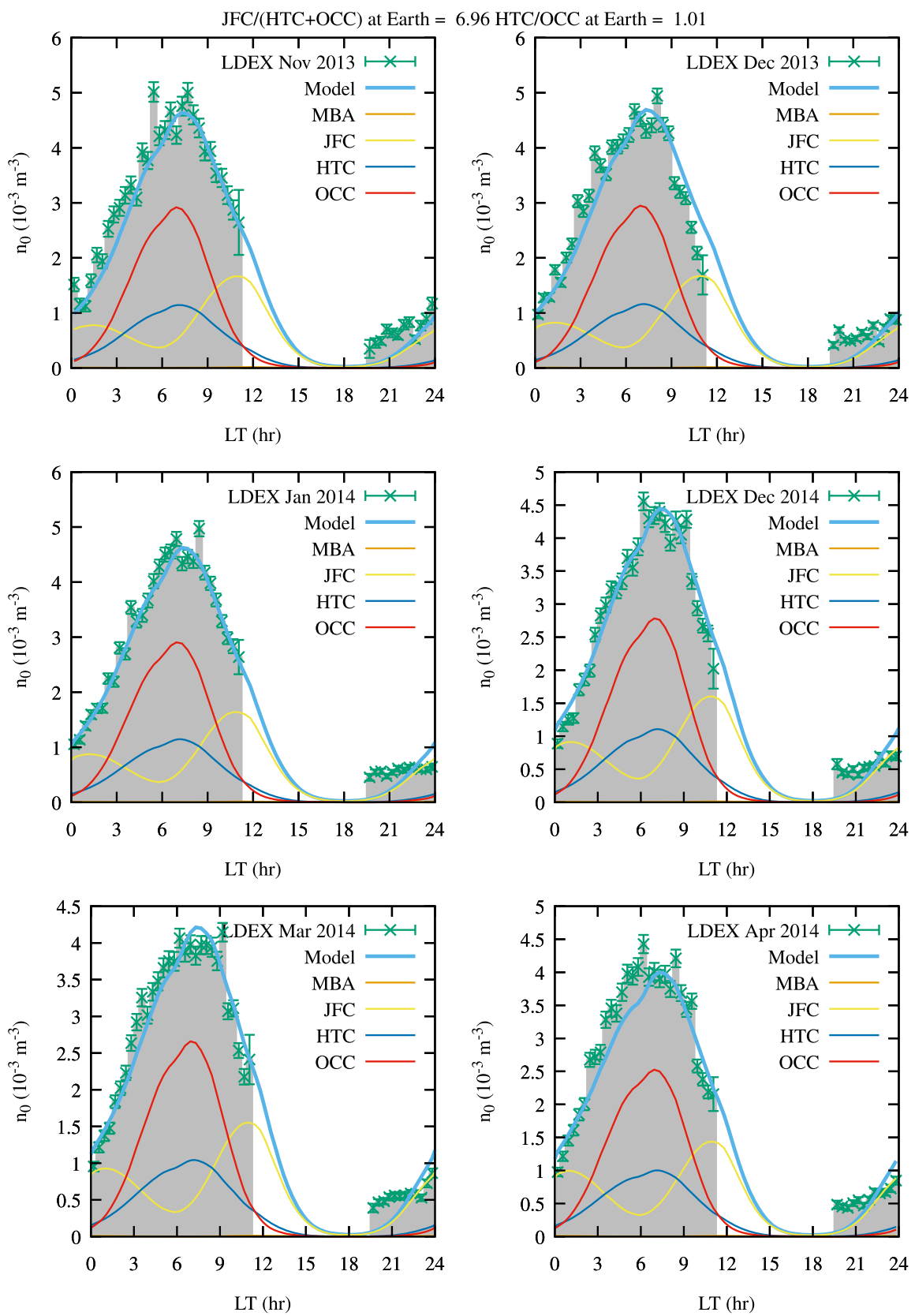

Fig. 10 LDEX data for November 2013-April 2014 (green points with error bars) compared to the model $M^{+}$reported by Pokorný et al. (2019), where the sum of the four populations is represented by the blue solid line. Contributions of individual meteoroid populations are represented by solid lines: MBA (orange), JFC (yellow), HTC (blue), OCC (red). A fit representing a JFC-to-LPC mass ratio at Earth equal to 6.99 was selected, and the HTC/OCC mass influx ratio at Earth equal to 1.04. The free parameters used for this figures are: $F_{\text {coll }}=20, \alpha=4, \gamma=1.23$. This is one out of many representations of the model with a similar goodness of fit $\left(\chi^{2}=7.99\right)$. The MBA contribution is negligible 
of LADEE/LDEX, the authors found that the predicted day-side values were systematically smaller than those measured by LDEX. A linear increase of ejecta mass production rate from $6 \mathrm{AM}$ to noon in lunar local time, convolved with the predicted meteoroid velocities and fluxes, provided significantly better agreement between the model and LDEX observations (Fig. 10) than assuming a similar response on the day and night sides. A different hypothesis was later proposed by Szalay et al. (2020b), who suggested that $\beta$-meteoroids (very small meteoroids on hyperbolic trajectories) hitting the Moon's sunward side could explain this asymmetry, since they can impact the Moon at very high speeds $\sim 100 \mathrm{~km} \mathrm{~s}^{-1}$ and thus their impact regime may differ from the significantly larger and slower sporadic meteoroids responsible for generating the bulk of the lunar impact ejecta cloud.

In addition to addressing the lunar day-side and night-side asymmetry, Pokorný et al. (2019) adopted the results reported by Carrillo-Sánchez et al. (2016) at Earth to estimate the absolute mass flux of meteoroids onto the Moon, similar to Mercury (Pokorný et al. 2018). The authors found that the total flux of MBA meteoroids cannot be constrained by modeling LDEX observations because they produce a negligible contribution to the total ejecta mass production rate due to their very low velocity. An important finding by the authors was that, in order to stay consistent with Earth-based estimates of the mass flux ratio of short-to-long period comets (Carrillo-Sánchez et al. 2016), the authors needed to revised the functional form of the ejecta mass production rate function, commonly used and suggested by Koschny and Grün (2001), finding that it should be linearly proportional to the meteoroid mass flux. Using constraints from Earth and taking into account the gravitational focusing effects between Earth and the Moon, Pokorný et al. (2019) finally concluded that the total mass accreted at the Moon is approximately $\mathcal{M}_{\text {Moon }}=1.4 \mathrm{t}_{\text {day }}{ }^{-1}$ assuming 43.3 $\mathrm{t} \mathrm{day}^{-1}$ at Earth, where the individual contribution of meteoroid populations are: JFCs $72.6 \%$, HTCs $\sim 12.8 \%$ and OCCs $\sim 10.0 \%$. An important note is that these results represent one of many possible fits to the available LDEX measurements and that the solution space to provide a similar or better fit is wide due to the limited selenographic coverage of LADEE.

Both Janches et al. (2018) and Pokorný et al. (2019) showed that JFCs meteoroids are concentrated close to the ecliptic plane, arriving from direction towards and away from the sun (helion/anti-helion sources). HTC and OCC meteoroids impact the Moon mainly towards the apex direction while MBA meteoroids have radiants ranging from all directions and are hence able to populate the anti-apex source. Like at Earth (Fig. 3), the apex source have average impact velocities exceeding $55 \mathrm{~km} \mathrm{~s}^{-1}$, while the toroidal and helion/antihelion sources are in general populated by meteoroids a factor of two slower. Due to the smaller gravitational focusing at the Moon, JFC and MBA meteoroids contribute 2.5 and 5 times less in terms of the mass flux to the lunar meteoroid environment, respectively, than at Earth. As a result of the broad latitudinal distribution of cometary impactors, the entire lunar surface can be exposed to impacts with velocities as high as $30 \mathrm{~km} \mathrm{~s}^{-1}$, where the near ecliptic directions can produce impacts with velocities up to $72 \mathrm{~km} \mathrm{~s}^{-1}$. This finding was later reiterated by Pokorný et al. (2020) who found that highly inclined orbits can easily access permanently shadowed regions and alter the surface properties via hypervelocity impacts even when the detailed topography of the lunar surface is taken into account.

Finally, Pokorný et al. (2019) showed that the meteoroid mass flux and, consequently, the impact vaporization flux and ejecta mass production rate experience yearly and monthly variations that can be well represented by a sum of two sine functions with periods of one year and 29.5 days (synodic period of the Moon, Fig. 11). Yearly mass flux variations amount to $3.3 \%$ of the yearly average mass flux, while monthly variations amount to only $0.2 \%$. These variations are larger for velocity dependent quantities, where yearly variations of the impact vaporization flux account for 6-8\%, while monthly variations are around $4-5 \%$. 




Day from July 1st, 2013

Fig. 11 Variations of the impact vaporization flux rate in $\mathrm{g} \cdot \mathrm{cm}^{-2} \cdot \mathrm{s}^{-1} \times 10^{-16}$. with time from 1 July 2013 to 30 June 2014. The thick black solid line shows a preferred solution, while the confidence interval is shown as the gray area between two black solid curves denoting minimum and maximum variations of the model. The orange thick line is a fit to our preferred solution by a sum of two sines. Two dashed vertical lines represent the time of aphelion (5 July 2013) and perihelion (4 January 2014) passage. From Pokorný et al. (2019)

When the full spectrum of impact velocities is taken into account, the apex/dawn terminator source is dominating both the impact vaporization flux and the ejecta mass production rate for any day of the year. The total vaporization rate was several times higher than estimated by Cremonese et al. (2013) due to the inclusion of LPC particles. This expected total vapor rate is higher than considered in lunar exosphere models (Sarantos et al. 2012b), meaning that the role of impact vaporization in supplying the lunar exosphere with metals may have been previously underestimated, especially for species like $\mathrm{Na}$ and $\mathrm{K}$ which do not condense.

\section{Meteoroid Influx on Other Inner Solar System Airless Bodies}

Impact ejecta production occurs on all airless bodies throughout the solar system. Unlike the Moon, which retains a large fraction of its secondary ejecta particles, small asteroids shed most their ejecta and contribute to the interplanetary dust population. These grains carry valuable information about the chemical composition of their parent bodies, which can be measured via in-situ dust detection (Cohen et al. 2019). The LADEE/LDEX measurements (Sect. 4) of the lunar dust cloud can be used to estimate the dust ejecta distribution for any airless body near 1 AU. As shown in Fig. 12, this dust distribution is expected to be highly asymmetric, due to non-isotropic impacting fluxes. Spacecraft flybys near these asteroids would experience many times more dust impacts by transiting the apex side of the body compared to its anti-apex side (Table 2). The ejecta cloud for airless bodies on eccentric orbits is more complex due to their radial velocities modulating the impact speeds of the 
Fig. 12 The predicted dust density distribution around asteroids on circular orbits near $1 \mathrm{AU}$ for grains with radii $a \geq 0.3 \mu \mathrm{m}$ is shown in the ecliptic frame for selected asteroid sizes. The scale of the dust cloud size is proportional to $R^{2}$, with $R$ the radius of the asteroid (Szalay and Horányi 2016b)

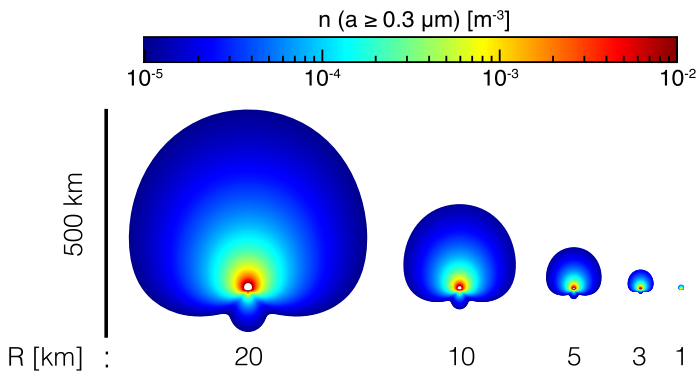

Table 2 Total number of predicted impacts per square meter during spacecraft flybys of an asteroid near $1 \mathrm{AU}$, with radius $R$ and closest approach distances $b=15,100 \mathrm{~km}$. The first column gives the assumed minimum detectable particle size. (Szalay and Horányi 2016b)

\begin{tabular}{llllll}
\hline$a_{\min }[\mu \mathrm{m}]$ & $R=1 \mathrm{~km}$ & & & $R=10 \mathrm{~km}$ & \\
\cline { 2 - 3 } & $b=15 \mathrm{~km}$ & $b=100 \mathrm{~km}$ & & $b=15 \mathrm{~km}$ & $b=100 \mathrm{~km}$ \\
\hline 0.1 & 20 & 2 & 2,000 & 200 \\
0.3 & 1 & $<1$ & 90 & 10 \\
1.0 & $\ll 1$ & $\ll 1$ & 3 & $<1$ \\
\hline
\end{tabular}

bombarding interplanetary dust particles. This is the case for the asteroid 3200 Phaethon

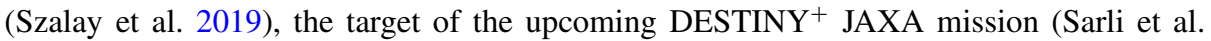
2018), which will carry a dust detector capable of chemical composition analysis (Krüger et al. 2019).

Recently, Szalay et al. (2019) used the same dynamical models utilized at the Moon by Janches et al. (2018) and Pokorný et al. $(2019,2020)$ to predict the morphology of a potential ejecta cloud around 3200 Phaethon, which should be highly asymmetric given the asteroid's high eccentricity. The authors found that at $1 \mathrm{AU}$, the cloud is canted towards the asteroid's apex direction and its density varies by five orders of magnitude. Furthermore, comparing the peak ejecta density to a body at the same heliocentric distance but with a circular orbit, 3200 Phaethon's peak ejecta cloud density is approximately 30 times higher, largely due to enhanced ejecta production from JFC meteoroids. This calculation implies that eccentric asteroids shed more material than those on near-circular orbits, and are thus more attractive candidates for in-situ dust detection and chemical characterization due to their amplified asymmetric ejecta production.

\section{Effects Due to Meteor Showers}

Interplanetary space is crisscrossed by streams of meteoroids with typical sizes in the hundreds of microns up to decimeters, which were liberated through the sublimation of cometary ices. Meteoroids are eventually scattered out of the stream due to gravitational perturbations by Jupiter and Saturn, becoming part of the sporadic background (Wiegert et al. 2009; Nesvorný et al. 2010). Initially, however, and for a period which may be as long as $10^{4}-10^{5} \mathrm{yr}$, the material remains confined to the streams, thus retaining a dynamical memory of its birth place. Unlike sporadics, meteoroids within streams move in approximately parallel paths and at nearly the same speed. The Earth intersects numerous streams 


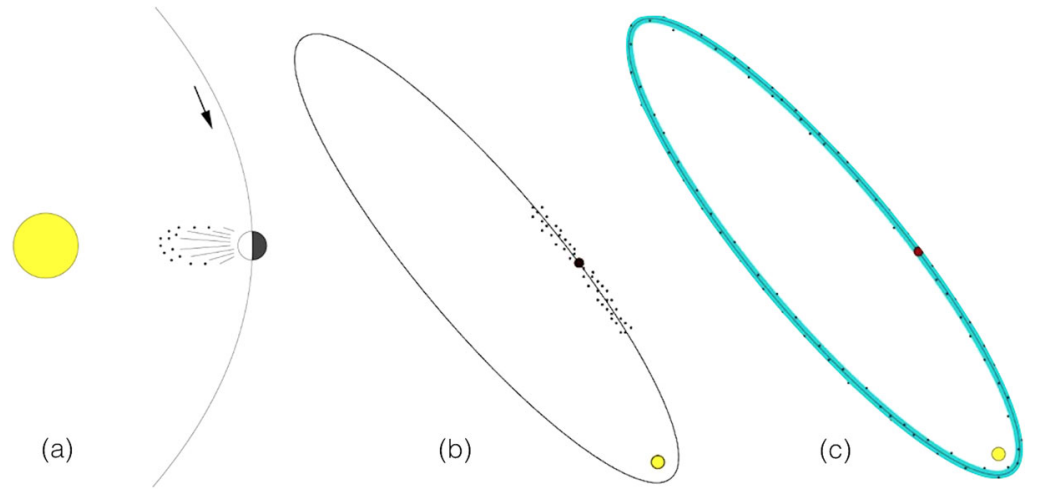

Fig. 13 Creation of a meteoroid stream. Meteoroids are ejected from a parent comet during a perihelion passage (a), initially forming azimuthally-confined trails of material along the orbit (b). Dust trails from different perihelion passages gradually spread out all around the orbit, forming the stream (c)

in its annual trek around the sun, resulting in periods of increased flux, or showers, of meteoroids at the top of the atmosphere (Brown et al. 2010; Pokorný et al. 2017a; Bruzzone et al. 2020). Although the SMC is responsible for providing a constant source of impactors to create and maintain exospheres on airless bodies, events produced by meteoroid streams offer a unique opportunity to study the physical processes involved in this phenomena with a much more constrained input (e.g., velocity distribution, directionality, temporal variability, etc).

Meteoroids newly ejected from the cometary nucleus (Fig. 13, panel a) initially form azimuthally-confined arcs of trails of particles (panel b) that persist over tens of orbital revolutions of the comet until incorporated into the stream by keplerian shear (panel c). Trails are responsible for meteor outbursts: intense, but short-lived, enhancements in the visible meteor rate at Earth, over and above the activity level of the annual shower (Janches et al. 2020 b). In many cases, the dynamical evolution of cometary trails is deterministic (Kondrateva and Reznikov 1985; McNaught and Asher 1999; Lyytinen and Van Flandern 2000; Vaubaillon et al. 2005) so meteor outbursts can be reliably forecasted with brute-force numerical simulations of test particles to serve as tracers of the dust evolution.

In contrast to Earth, there is precious little information on meteoroid streams incident on other planetary bodies. In principle, every planet-approaching comet contributes some amount of dust to the local meteoroid environment. In practice, the dynamical mobility and dust properties of the comet will determine the magnitude of its contribution to the flux. The complex relationship between cometary activity and the planet-intersecting component of the dust means that - even for the Earth - it is practically impossible to make reliable quantitative predictions of the flux, except in cases where the dust has been detected previously in the planetary vicinity, for example as a meteor shower. A case in point is the exceptionally close $(\sim 150,000 \mathrm{~km})$ approach of comet C/2013 A1 (Siding Spring) to Mars in October 2014. Despite intense scrutiny of the comet in the year leading up to the encounter, predictions for a meteor shower in Mars's atmosphere ranged from storm-level $\left(10^{7} \mathrm{~kg}\right.$ of dust mass deposited in the atmosphere; Vaubaillon et al. 2014) to negligible ( $<100 \mathrm{~kg}$ deposited; Kelley et al. 2014). Post-encounter estimates inferred from observations of metallic species in the upper atmosphere were closer to the upper end of predictions (Schneider et al. 2015). 


\subsection{Meteoroid Streams at the Moon}

The Moon is a unique natural laboratory for understanding the response of an airless body to passage through a stream and has important lessons to teach us with respect to Mercury. The Moon's proximity to the Earth means that the two bodies share a common meteoroid environment and, because meteor showers are known from terrestrial observations, lunar measurements can be readily linked to showers. Indeed, seismic data from the Apollo Passive Seismic Experiment detector network has shown the lunar impact rate to be highly nonrandom. Several impact event clusters identified in the seismic data correspond to strong Earth meteor showers (Oberst and Nakamura 1991). One remarkably dense swarm of meteoroids detected by the Apollo network in 1975 (Duennebier et al. 1976) was associated with the so-called Taurid complex, to which we shall return to later.

Flashes caused by kg-class meteoroids impacting the lunar surface were first detected over two decades ago (see Madiedo et al. 2019, for a review on this topic). Early observations, focused on maximising the impact detection rate, were preferentially carried out during meteor showers. These were followed by surveys where observations took place regularly under favourable lunar observing conditions, showing that the occurence of flashes is indeed correlated with showers (Suggs et al. 2014; Liakos et al. 2020). After the impact of a kg-sized meteoroid near the lunar terminator two dust clouds with typical expansion velocities of 0.1 and $3 \mathrm{~km} \mathrm{~s}^{-1}$ were observed (Berezhnoy et al. 2019). The most recent observations of impact flashes detect impactors as small as $1 \mathrm{gr}$ and about $1 \mathrm{~cm}$ in size (Avdellidou and Vaubaillon 2019). The derived temperatures from these flashes are consistent with hypervelocity impact experiments (Eichhorn 1975). These flashes not only provide microphysical parameters for exosphere simulations, but also produce stochastic variation in the exosphere (Mangano et al. 2007).

The response of the lunar exosphere to meteoroid bombardment by meteoroid shower activity has been identified in the past. Reports by Verani et al. (1998, 2001), Smith et al. (1999) and Berezhnoy et al. (2014) observed various degrees of exospheric increase of sodium at the Moon during the Leonids, Taurids, Quadrantids, and Perseids. As stated in Sect. 4, LADEE's observations were instrumental at providing detailed measurements of the effects of meteoroids in general and showers in particular on the lunar surface. LDEX detected episodes of enhanced flux of lunar ejecta coinciding with known meteor showers (See Fig. 8 and Szalay and Horányi 2015). The high temporal cadence of LADEE measurements permitted detailed studies of shower effects. For example, Szalay et al. (2018) reported a large enhancement in the lunar impact ejecta cloud while the Moon transited the Geminid meteoroid stream, particularly above the portion of lunar surface normal to the shower mean radiant. The authors found two peaks in the estimated surface density of impact ejecta which coincided with radar observations of shallower mass indices than most of the Geminids, suggesting an enhancement of larger particles. The timing of the main observed peak matched groundbased visual observations of meteors with magnitude of -1 to -3 . This finding suggests that LDEX detected ejecta from primary impactors with radii $\sim 2 \mathrm{~mm}$ to $2 \mathrm{~cm}$.

LADEE was the first mission to directly observe the link between meteoroid bombardment and exosphere formation (Elphic et al. 2014) because, in addition to the LDEX dust experiment, it also carried an Ultraviolet-Visible Spectrometer (UVS; Colaprete et al. 2014) and a Neutral Mass Spectrometer (NMS; Mahaffy et al. 2014). The comparison between the observations of LDEX and UVS identified a correlation between the meteoroid influx and the $\mathrm{Na}$ and $\mathrm{K}$ abundances in the lunar exosphere, in particular with shower activity. Specifically, Colaprete et al. (2016) and Szalay et al. (2016) found a strong correlation of exospheric potassium and meteoroid ejecta during the Geminids meteoroid shower, exhibiting a much stronger response than sodium. With the exception of the Geminids, the authors 
Fig. 14 Cumulative number of comets as a function of $q$, the perihelion distance. Comets with $q<0.05 \mathrm{AU}$ are not counted. The horizonal segments represent the annual radial excursions of planetary orbits and have been shifted vertically to intersect the curve

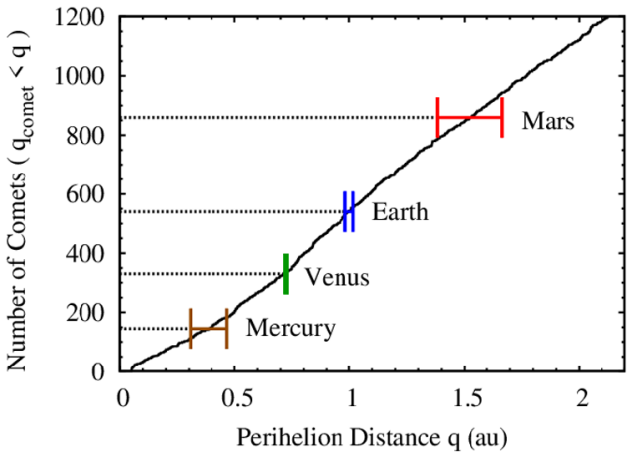

found a weak correlation between the meteoroid influx as measured by LDEX and exospheric density of alkalis as measured by UVS. Similarly with NMS, Benna et al. (2019) reported detections of water vapor released into the lunar exosphere. The timing of 29 water release events agreed with periods when the Moon encountered known meteoroid streams. The authors used these measurements to constrain the hydration state of the lunar soil, arguing that by heating the soil meteoroids release water that is buried below a layer of dry regolith at depths of a few centimeters.

\subsection{Meteoroid Streams at Mercury}

The meteoroid stream environment at Mercury is effectively unconstrained by direct observation and, at least for the time being, arguments for or against their existence must be based on indirect evidence. Since most known streams are associated with active comets, useful conclusions may be drawn by comparing the number of comets approaching Mercury and the Earth. A necessary condition for two orbits to cross is

$$
q_{\text {Comet }}<Q_{\text {Planet }}
$$

where $q_{\text {Comet }}$ is the perihelion distance of the comet and $Q_{\text {Planet }}$ the planet's aphelion distance. For a near-circular orbit, $Q$ can be replaced by the semimajor axis $a$. This is not a sufficient condition because, whether the orbits actually cross depends on the relative geometry between the orbits. Under the working assumption that orbit orientation is - at least within the terrestrial planet region - insensitive to heliocentric distance, examination of the distribution of cometary $q$ should constrain the relative number of streams at Mercury and the Earth.

For this purpose, works that deal with meteor showers in the solar system (e.g., Selsis et al. 2004) utilize the DASTCOM database (https://ssd.jpl.nasa.gov/?sb_elem, retrieved 09 May 2020) available through the JPL online solar system data service (Giorgini et al. 1996). This set contains several clusters of comet fragments sharing the same orbit and, in addition, many sungrazing comets, where $q_{\text {Comet }}$ is comparable to the solar radius. Including these clusters would skew the statistics, therefore all but one of the fragment orbits in each cluster were removed. Most sungrazers have $q<0.05$ AU (Wiegert et al. 2020) so all comets with $q$ below that value are removed.

Figure 14 shows the cumulative distribution of $q, N(q)$, for the DASTCOM entries. The horizontal segments correspond to the radial excursions of the four terrestrial planets. It can be seen in this figure that each of the planetary orbits encompasses the perihelia of 860 (Mars), 540 (Earth) \& 330 (Venus) comets while the figure for Mercury is 150 . Taken these 
numbers at face value suggests that Mercury encounters $1 / 3$ to $1 / 4$ of number of streams that would encounter Earth in its annual trek around the Sun. Strictly speaking, this result only applies to comets near the ecliptic plane where the perihelion criterion (Eq. (1)) automatically ensures a close approach. For an isotropic comet population, the distribution should be adjusted for the reduced volume of available heliocentric space enclosed by Mercury's orbit relative to the Earth but also the tendency for stream cross sections to be minimized near perihelion where most stream meteoroids were ejected. Christou (2004) computed Minimum Orbit Intersection Distances (MOIDs) for a sample of 158 multi-apparition comets in DASTCOM. The number of comets with orbital period $P<20 \mathrm{yr}$ approaching Mars, Earth and Venus to $<0.1 \mathrm{AU}$ was reported to be 31,12 and 4 for those three planets, in qualitative agreement with Fig. 14. The respective figures for comets with $P>20 \mathrm{yr}$ were 3, 3 and 5. Small number statistics notwithstanding, it is reasonable to expect that the number of isotropic comet streams approaching Mercury and the Earth are similar.

Numerical modelling of individual streams is motivated by the need to explain observations (e.g., the Leonid storms; McNaught and Asher 1999). For Mercury, the seasonal modulation of exospheric Ca observed in situ by MESSENGER (Fig. 6 and Burger et al. 2014) motivated modelling of the sporadic background (Killen and Hahn 2015; Pokorný et al. 2018) to investigate meteoroid surface impact vaporization as the source mechanism. As seen in Fig. 6 on this chapter, these models reproduce the overall Ca production rate dependence with TAA but fail to predict a positive feature at TAA $=20-30^{\circ}$. It was suggested by Killen and Hahn (2015) that the cause may be due to an enhanced bombardement of meteoroids by a stream originated from comet 2P/Encke, although this comet crosses Mercury's orbit plane further away from perihelion, at TAA $=45^{\circ}$. Encke has been linked to several strong daytime and nighttime meteor showers at Earth, the so-called Taurid complex (Whipple 1940; Porubčan et al. 2006). Its current orbit is $~ 0.17$ AU from the Earth's but only 0.026 AU from that of Mercury (Selsis et al. 2004). Recently, Stenborg et al. (2018) detected reflected light from the Encke stream near Mercury's orbit in SECCHI/STEREO images.

Christou et al. (2015) investigated the properties of the near-Mercury Encke stream with a two-parameter numerical model. One parameter was age (i.e., the time spent by meteoroids in space since ejection from the nucleus); the other was the particle size, which determines $\beta$ the strength of solar radiation forces relative to gravity on the meteoroid through the expression

$$
\beta=1150(\rho D)^{-1}
$$

where $\rho$ in this context is the bulk density in $\mathrm{kg} \mathrm{m}^{-3}$ and $D$ is the diameter in $\mu \mathrm{m}$.

It was found that, despite the proximity of the orbits, Encke-released dust younger than 5 kyr has not undergone sufficient orbital evolution to physically reach Mercury. At the same time, planetary gravitational effects begin to disperse the stream after $\sim 50 \mathrm{kyr}$. PR drag plays a crucial role in delivering Encke released dust to Mercury by rotating the line of nodes of stream meteoroids away from the comet's (Fig. 15). Indeed, an optimum combination of size and age exists for the orbits to cross Mercury's, at TAA of $350-30^{\circ}$; these are meteoroids ejected 10-20 kyr ago with $\beta=1-210^{-3}$, equivalent to particles a few $\mathrm{mm}$ in size for $\rho=600 \mathrm{~kg} \mathrm{~m}^{-3}$ (Eq. (2)). The particles will arrive at Mercury from the antisolar direction and impact on the nightside at $32-37 \mathrm{~km} \mathrm{~s}^{-1}$. The simulations also showed that the stream intersects Mercury's orbit a second time, at TAA $=135^{\circ}-165^{\circ}$, with the meteoroids impacting on the dayside and on the outbound leg of their heliocentric trajectories. Christou et al. (2015) reported a minor enhancement in the Ca production rate profile at TAA $\sim 165^{\circ}$ 


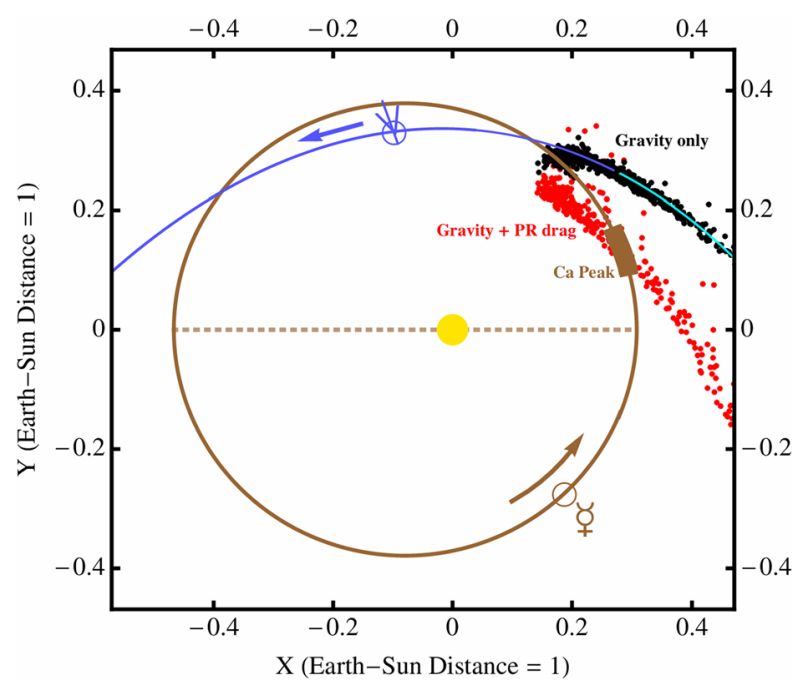

Fig. 15 Locations of descending nodes of $10^{4}$ yr-old Encke particles on Mercury's orbit plane at JD2487500.0. The comet orbit projection on this plane is represented by the blue curve with the part of the orbit lying above the plane shown in a brighter colour. Mercury's orbit is represented by the brown ellipse with a dashed line connecting the orbit apses. The yellow point represents the Sun. The brown rectangle represents the location of the $\mathrm{TAA}=25^{\circ}$ peak in Calcium production rate inferred from MESSENGER observations. Black points correspond to particles subject to planetary gravitational perturbations only; red points are affected by Poynting-Robertson drag with $\beta=10^{-3}$ in addition to gravity

that may be seen to corroborate this second crossing, yet the evidence is less conclusive than for the peak at TAA $=25^{\circ}$.

\section{Laboratory Experiments}

Hypervelocity dust impact experiments can be used to establish the efficiency of the production of neutrals and ions by the continual bombardment of the lunar surface by meteoroids. For example, Sugita et al. $(1998,2003)$ conducted experiments at the NASA Ames Vertical Range using spherical copper projectiles and polycrystalline dolomite targets to record the intensities of emerging atomic lines and molecular bands. The measured emission intensities as function of the speed and mass of the impactor suggested that the impact-vapor contribution to the lunar exosphere involves a complex chain of chemical and physical processes. The first direct laboratory measurement of vapor produced by bombardment with simulated micrometeoroids in the size range of $0.1-1 \mu \mathrm{m}$ radius and speed range of $1-$ $10 \mathrm{~km} \mathrm{~s}^{-1}$, used a fast ion gauge (Fig. 16) to quantity the neutrals released per unit projectile mass, $N / m$ (Collette et al. 2014). The results indicated a power-law dependence with the projectile speed $v$, as $N / m \sim v^{2.4}$ (Fig. 17). At the highest speeds tested, the number of neutral atoms liberated is equivalent to $5 \%$ of the atoms in the projectile; complete vaporization is expected at speeds exceeding $20 \mathrm{~km} \mathrm{~s}^{-1}$. Earlier experiments (Eichhorn 1975) had established the expected temperature of the impact-generated clouds.

At the Moon, the meteoritic source contribution to sustain the dilute exosphere competes with solar wind sputtering (for refractory metals) and photon-stimulated desorption, or PSD (for alkalis). For example, Stern (1999) estimated a total exospheric mass of about 
Fig. 16 Schematics of the hot-filament ionization gauge used to detect the pressure increase caused by a dust impact. From Collette et al. (2014)
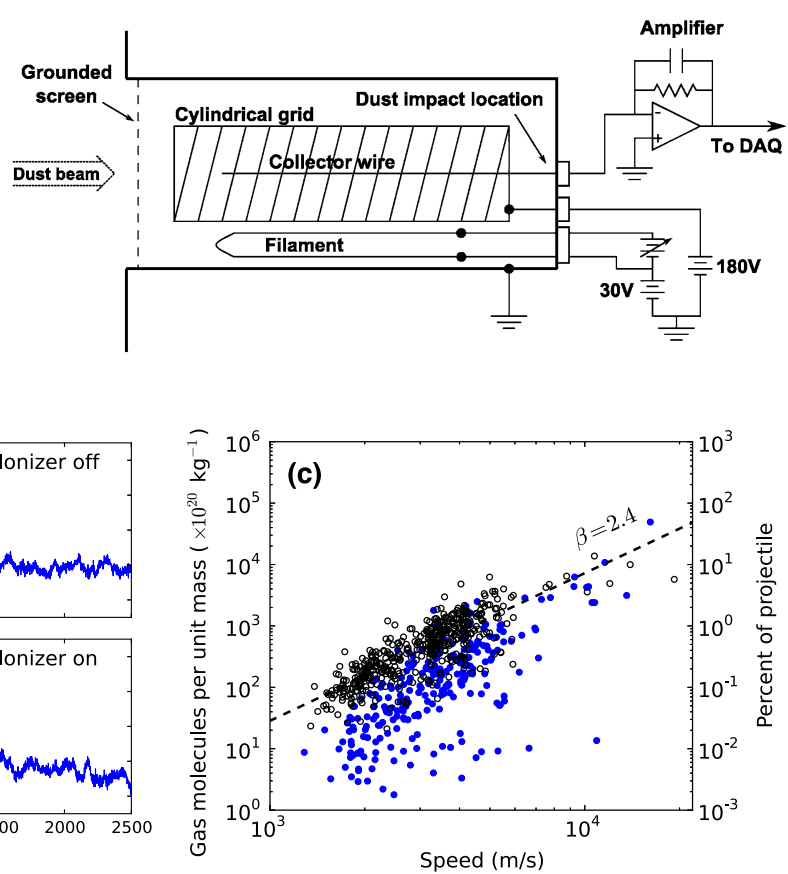

Fig. 17 The recorded ion gauge (Fig. 16) signals with and without the ionizer turned on for two similar dust particle events: (a) $m=2.3 \times 10^{-12} \mathrm{~g}, v=3.2 \mathrm{~km} \mathrm{~s}^{-1}$; and (b) $m=2.0 \times 10^{12}, v=3.5 \mathrm{~km} \mathrm{~s}^{-1}$. (c) The amount of neutral gas molecules recorded (left axis) as function of the dust particle's impact speed with the ionizer on (open black circles) and off (closed blue circles), and the number of neutral atoms as the fraction of the atoms in the projectile (right axis). From Collette et al. (2014)

$2 \times 10^{7} \mathrm{~g}$, asuming that approximately 5 tons/day $(60 \mathrm{~g} / \mathrm{s})$ of interplanetary sub-milligram sized meteoroids bombard the lunar surface, about $\sim 2.5$ time more mass that recent estimates (Pokorný et al. 2019). The authors argued that the total mass of incoming meteoroids delivered in about 4 days is comparable to the mass of the entire lunar exosphere. The importance of meteoroids increases for less volatile metals such as $\mathrm{Mg}, \mathrm{Ca}, \mathrm{Al}$, and others, and it is expected to provide about half of the total abundance (Sarantos et al. 2012a).

\section{Large Impactors}

As described above, much of the scientific research the meteoroid impact effects on an exosphere, and the focus of this manuscript, has been in regards to very small objects diameters smaller than a millimeter, which impact the surface often enough to form and constantly maintain the exosphere (See Sect. 2). As discussed in the earlier sections, the orbital characteristics of meteoroids smaller than this, have evolved dynamically due to various effects. In this section we focus on the rarer impacts made by meteoroids that are much larger in size. As it is the case for meteor showers, when detected, large impactors have a well defined set of physical and dynamical characteristics and thus can help to understand the associated exospheric processes more accurately.

The differential radius distribution, $h(r)$, for impactors in the size range from $1 \mathrm{~cm}$ to $100 \mathrm{~m}$ is shown in Fig. 18 (Marchi et al. 2005). This shows the number of impacts per 
Fig. 18 Differential radius distribution, $h(r)$, which shows the number of impacts for the whole surface per year and size in meters, for Earth and Mercury. From Marchi et al. (2005)

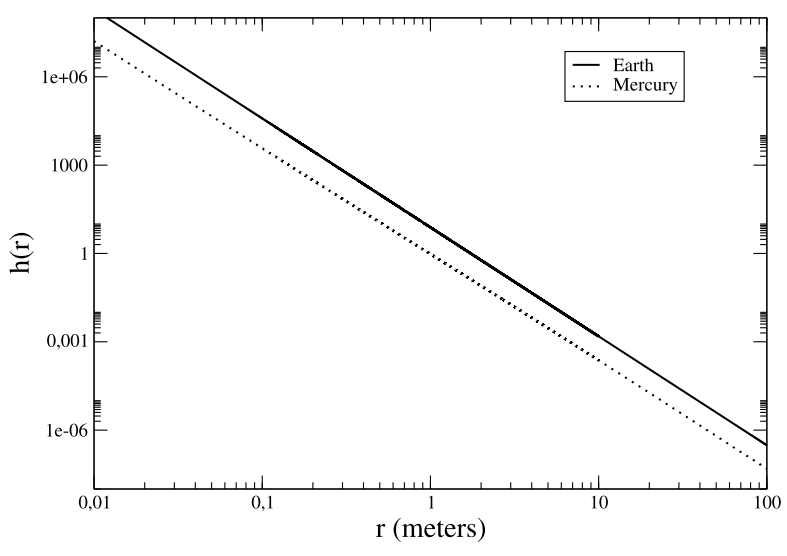

year and size for both Earth and Mercury as a function of radius. It can be seen that the expected rate of impacts decreases with the size of the impactor, being the impact rate of $1 \mathrm{~m}$ size meteor of 1 per Earth-year at Mercury, that is, one order-of-magnitude lower than at Earth. These larger meteoroids have a different dynamical evolution and arrive from the asteroid belt due to the 3:1 and $v_{6}$ resonances. The 3:1 resonance lies approximately at 2.5 $\mathrm{AU}$, and objects undergo a change in their eccentricity until they become Earth or Mars orbit-crossing. At this point they can be extracted from the asteroid belt. The $v_{6}$ resonance occurs when the object's longitude of perihelion precession frequency is equal to the sixth secular frequency of the planetary system (for asteroids, this is usually Saturn; Morbidelli and Gladman 1998). This resonance forces the objects to cross the orbits or Mars, Earth or Venus as well as collide with the Sun. These two resonances therefore act to eject objects from the asteroid belt into the inner solar system where they can impact on planetary bodies such as Mercury or the Moon. A study of 59 meter-sized Earth-impactors found that the $v_{6}$ resonance dominates the delivery mechanism with $50 \%$ of the probability that the impactors originated from the asteroid belt (Brown et al. 2016). These types of large impacts are rare in comparison to the much smaller scale meteroids (Pokorný et al. 2018, 2019), and are expected to contribute only transient changes to the exospheres of Moon and Mercury.

Figure 19 shows the observations of an impact flash on the Moon on March 17, 2013. The impactor was estimated to have a diameter of 9-15 $\mathrm{m}$, a mass of $16 \mathrm{~kg}$ and produced a crater with an estimated rim-to-rim size of 12-20 m (Suggs et al. 2014). The authors analysed a dataset of 126 other observed impact flashes at the Moon in 2006-2011, with impactors ranging in size from $1 \mathrm{~cm}$ to $14 \mathrm{~cm}$.

Mangano et al. (2007) modelled the effect of similar impactors at Mercury. They investigated how an impact would generate an enhancement of the local exosphere at altitudes of $400 \mathrm{~km}$ and $1500 \mathrm{~km}$, for the most common constituents of Mercury's surface composition. The model showed that the largest density enhancements at $1500 \mathrm{~km}$ above the average exospheric density would be for $\mathrm{Mg}, \mathrm{Si}$ and $\mathrm{Al}$. However, such enhancements are expected to be short-lived, with local enhancements falling back to average densities in timescales of less than an hour.

Such an enhancement was observed by the MESSENGER Fast-Imaging Spectrometer, which measured freshly ionized pick-up ions in the solar wind at high altitudes (Jasinski et al. 2020). The ions were estimated to come from a neutral "plume" of impact vaporized surface material most likely caused by a $1 \mathrm{~m}$ sized meteoroid. The neutrals were then photoionized and the ions were picked up by the local solar wind plasma and subsequently 


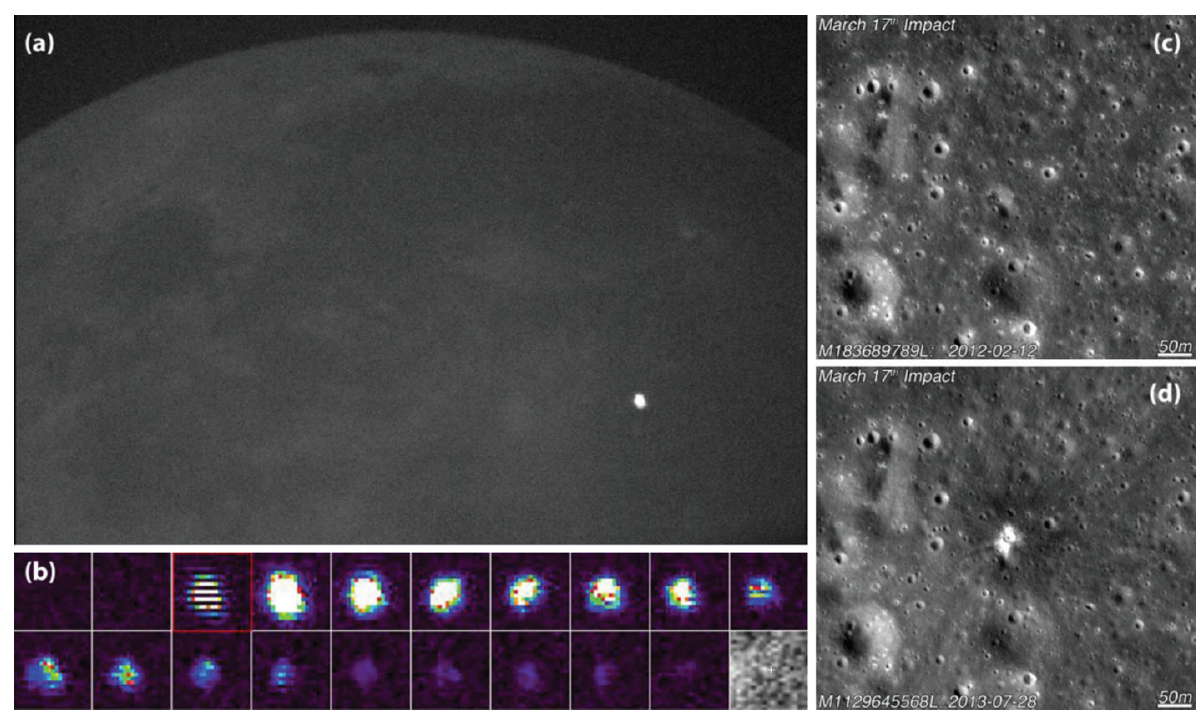

Fig. 19 Images from an impact on the Moon which was observed on March 17, 2013 Suggs et al. (2014). Panel (a) the full field of view of the observed flash, (b) images taken from the 30 FPS video of the impact flash, (c) and (d) the before and after images of the impact site observed by the Lunar Reconnaissance Orbiter Camera. From Suggs et al. (2014)

observed spacecraft's Fast-Imaging Plasma Spectrometer. A schematic of the process can be seen in Fig. 20; the Sun is to the left of Mercury, and the magnetospheric boundaries are shown (white curved lines). The orientation of the interplanetary magnetic field and the motional electromagnetic field are shown for the observed event in the top left corner. The pickup ions were measured to be within a mass-per-charge ratio of 21-30 amu/e, which includes $\mathrm{Na}^{+}, \mathrm{Mg}^{+}, \mathrm{Al}^{+}$, and $\mathrm{Si}^{+}$. Due to the long and short photoionization lifetimes of $\mathrm{Mg}$ and $\mathrm{Al}$, respectively, the composition of the ions from the impactor "plume" was estimated to be primarily of $\mathrm{Na}^{+}$and $\mathrm{Si}^{+}$. BepiColombo, an ESA and JAXA mission with two spacecrafts that will orbit Mercury, is expected to observe more of such events (at the time of writing BepiColombo is in its cruise phase with orbit insertion expected in 2025).

\section{Future of the Field}

Over the upcoming decade, there are multiple opportunities for spaceborne dust measurements at $1 \mathrm{AU}$ and in the inner Solar System. The DESTINY ${ }^{+}$(Demonstration and Experiment of Space Technology for INterplanetary voYage Phaethon fLyby dUSt science; Krüger et al. 2019) and IMAP (Interstellar Mapping and Acceleration Probe; McComas et al. 2018) missions, which will be launched in 2024, carry dust analyzers with the ability to perform chemical composition analysis of interplanetary dust and/or interstellar dust at $1 \mathrm{AU}$. Besides interplanetary and interstellar dust measurements around $1 \mathrm{AU}, \mathrm{DESTINY}^{+}$will also observe the ejecta cloud around the active asteroid 3200 Phaethon for the first time allowing us to constrain our current predictions (Szalay et al. 2019). IMAP will focus on the observation of interstellar dust, potentially allowing to link the composition of solar wind ions with the constituents of interstellar dust. The Tanpopo experiment, a sample return mission with a silica aerogel capture medium on the International Space Station, will provide the 


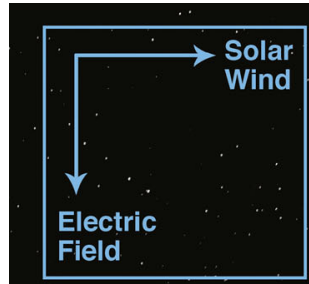

Magnetic Field Direction

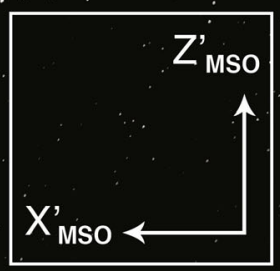

OY'MSO

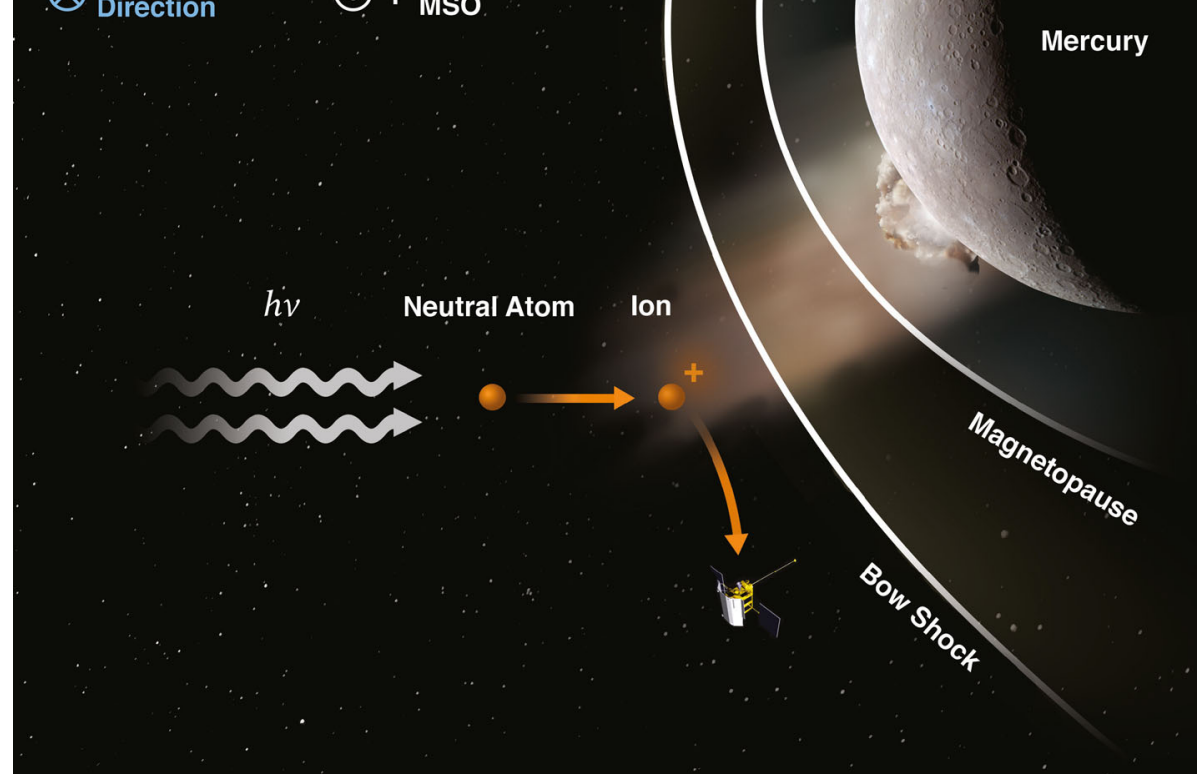

Fig. 20 A schematic from Jasinski et al. (2020) showing the photoionization of neutral particles released from the surface of Mercury due to a large impactor. The newly photoionized particles were observed as pickup ions by the MESSENGER spacecraft in the solar wind upstream of the bow shock

information of chemical composition of dust particles falling onto the Earth (Tabata et al. 2016). For the next space station orbiting around the Moon, the Lunar Orbital PlatformGateway, a number of international contributions for dust measurements are expected that, taking advantage of the long duration and large infrastructure of this platform, can lead to better understanding of the Earth-Moon meteoroid environment. In contrast to the larger space missions, $6 \mathrm{U}$-class $(10 \mathrm{~cm} \times 20 \mathrm{~cm} \times 30 \mathrm{~cm})$ deep-space explorer, EQUULEUS, will detect the first dust impact by a CubeSat during the cruise to a libration orbit around the Earth-Moon Lagrangian point L2 (Funase et al. 2020). In the innermost region of our Solar System, the Parker Solar Probe instrumentation has already reported impacts by very small dust particles which seem to be consistent with the existence of a dust population on hyperbolic trajectories, i.e. $\beta$-meteoroids (Mann et al. 2019; Szalay et al. 2020a). Although the mass influx of these dust particles represent, most likely, a minor contribution to the formation of exosphere of airless bodies, it could be responsible for some effects such as the night-day asymmetry observed by LADEE on the lunar dust cloud (Szalay et al. 2020b) and they can impose constraints on the size distribution of mass-dominant meteoroids (Fig. 1). LADEE findings provide a unique opportunity to map the composition of the lunar surface 
from orbit (Postberg et al. 2011) and identify regions that are rich in volatiles, providing opportunities for future in situ resource utilization (ISRU) (Horányi et al. 2020)

Clearly one of the most exciting opportunities to continue growth in this field is the arrival of the dual-spacecraft ESA-JAXA Bepicolombo mission at Mercury in 2025, which will provide simultaneous observations of meteoroid impact, exospheric neutrals and ions in the Hermean orbit. These observations will set more constraints on models described in this manuscript as well as test the link between Mercury's exosphere and the Encke meteoroid stream (Plainaki et al. 2017). The most direct evidence for temporal dust flux enhancements indicative of a stream would likely come from the Mercury Dust Monitor (Nogami et al. 2010) onboard the Mercury Magnetospheric Orbiter module. This instrument should register ejecta from the surface impact of Encke meteoroids, similarly to LADEE on the Moon (Szalay and Horányi 2016). Concurrent exosphere measurements by the SERENA and PHEBUS (Quémerais et al. 2020) instruments onboard the Mercury Planetary Orbiter (MPO) module and MSASI on the Magnetospheric Orbiter would enable establishing a relationship, if any, between $\mathrm{Ca}, \mathrm{Na}$ or other exospheric species and meteoroid impacts. An exciting possibility is the direct detection of impact flashes by stream meteoroids, despite the short-lived and upredictable nature of the phenomenon. The observation of the flashes will represent a serendipity project of SIMBIO-SYS (Cremonese et al. 2020), the suite of cameras on board the MPO, when it will observe the dark hemisphere of Mercury for calibration purposes.

Another area which requires further work concerns laboratory experiments. In the last few years significant progress has been made regarding the physical processes that meteoroids undergo upon atmospheric entry (i.e. ablation and ionization; Thomas et al. 2016; DeLuca et al. 2018; Gómez Martín et al. 2017; Bones et al. 2016). However, there are still major unknowns regarding meteoroid impacts on planetary surfaces. As described in Sect. 4, current models utilized experimental results from Koschny and Grün (2001) in order to obtain absolute values of the impact mass production rates $M^{+}$for the lunar surface, which yield a proxy of the gardening rate from meteoroids. Current modeling results suggest that equatorial regions experience three to five times higher ejecta production - and thus gardening - rates than the polar regions. They also predict lunar dust cloud density values higher by four orders of magnitude than those inferred by LDEX (Pokorný et al. 2019). This discrepancy indicates that the ejecta mass production yield of lunar regolith is considerably lower than the experimental data reported by Koschny and Grün (2001) yield which was estimated using very low velocity impacts $\left(1-12 \mathrm{~km} \mathrm{~s}^{-1}\right)$ on ice-rich surfaces. Clearly better designed experiments are needed to advance in this area.

Finally, models are constantly updated as new data almost always provide new paradigms. An important point to raise is that current meteoroid models use the ecliptic plane as a reference plane rather than, for example, the invariable plane of the solar system. For instance, Cambioni and Malhotra (2018) reported that the main asteroid belt is inclined by about a degree relative to the ecliptic. If the sporadic meteoroid complex is symmetric about the invariable plane (or some other plane) rather than the ecliptic, it could significantly change the presented results. It is very likely that the sporadic meteoroid complex is warped or twisted and the plane of symmetry changes between the main belt and the innermost parts of the solar system. For example Nesvorný et al. (2006) treated the dust bands in the main-belt as symmetric about the invariable plane when looking outward. On the other hand, Nesvorný et al. (2010) assumed that the ZDC is symmetric with respect to the ecliptic latitude when trying to reproduce IRAS measurements. Rowan-Robinson and May (2013) also showed that the ecliptic plane works well as the symmetry plane for the ZDC. However, between 0.3 and $1 \mathrm{AU}$ the plane of symmetry seems to be different from the invariable plane and similar to the orbital plane of Venus according to Leinert et al. (1980). 
There are several reasons why the assumption of ecliptic symmetry in the dynamical models presented here makes sense. Initially, some of these models were constrained with IRAS measurements, which showed that the Zodiacal cloud is symmetric with respect to the ecliptic (Nesvorný et al. 2010, 2011a,b). This conclusion is also supported by decades of observations using meteor radars which have shown that, as viewed from Earth, the main sporadic sources are symmetric with respect to the ecliptic plane (Fig. 2; Janches et al. 2015; Campbell-Brown and Wiegert 2009). Later, the HTC dynamical model reported by Pokorný et al. (2014) reproduced radar observations assuming ecliptic symmetry. Furthermore, earlier semi-empirical models also concluded that a meteoroid environment that is symmetric with respect to the ecliptic plane reproduced the seasonality and geographical variability of meteor observations using high power and large aperture radars (Janches et al. 2006; Fentzke and Janches 2008; Fentzke et al. 2009; Pifko et al. 2013; Schult et al. 2017). Finally, ecliptic symmetry seems to reproduce, at least to first order, the variabilities observed on the lunar dust cloud (Janches et al. 2018; Pokorný et al. 2019) and the Ca exosphere at Mercury (Pokorný et al. 2018).

It is important to note that most of these works concern meteoroids with cometary origin which have enough energy to produce the reported observed and modeled phenomena, while Cambioni and Malhotra (2018) focused on the MBA population. Meteoroids originating from MBAs are too slow to produce ionization (and thus most of the observed radar meteors), ablate in the atmosphere and produce metallic layers, or even produce a significant contribution to the dust plumes in airless bodies. The Zodiacal Cloud is certainly more complex than current models predict and investigating such effects offers exciting future opportunities.

Acknowledgements DJ and MS were funded through the NASA ISFM and LDAP Programs. A.A.B. was partially supported by Russian Science Foundation, grant no. 20-12-00105. GC contribution is supported by the ASI-INAF agreement 2017-47-H.0. JMJ's contribution to this work was supported by an appointment to the NASA Postdoctoral Program (NPP) Fellowship at the Jet Propulsion Laboratory administered by the Universities Space Research Association through a contract with the National Aeronautics and Space Administration (NASA). Astronomical research at the Armagh Observatory and Planetarium is grant-aided by the Northern Ireland Department for Communities (DfC).

Funding Note Open access funding provided by Istituto Nazionale di Astrofisica within the CRUI-CARE Agreement.

Open Access This article is licensed under a Creative Commons Attribution 4.0 International License, which permits use, sharing, adaptation, distribution and reproduction in any medium or format, as long as you give appropriate credit to the original author(s) and the source, provide a link to the Creative Commons licence, and indicate if changes were made. The images or other third party material in this article are included in the article's Creative Commons licence, unless indicated otherwise in a credit line to the material. If material is not included in the article's Creative Commons licence and your intended use is not permitted by statutory regulation or exceeds the permitted use, you will need to obtain permission directly from the copyright holder. To view a copy of this licence, visit http://creativecommons.org/licenses/by/4.0/.

\section{References}

P.A.R. Ade, N. Aghanim, C. Armitage-Caplan, M. Arnaud, M. Ashdown, F. Atrio-Barandela, J. Aumont, C. Baccigalupi, J. Banday Astron et al., Planck 2013 results. XIV. Zodiacal emission. Astron. Astrophys. 571, A14 (2014). https://doi.org/10.1051/0004-6361/201321562. arXiv:1303.5074

W.M. Alexander, O.E. Berg, C.W. McCracken, L. Secretan, J.L. Bohn, Interplanetary dust-particle flux measurements between 1.0 and 1.56 a. u. from Mariner 4 cosmic-dust experiment (abstract). Smithson. Contrib. Astrophys. 11, 227 (1967) 
C. Avdellidou, J. Vaubaillon, Temperatures of lunar impact flashes: mass and size distribution of small impactors hitting the Moon. Mon. Not. R. Astron. Soc. 484(4), 5212-5222 (2019). https://doi.org/10.1093/ mnras/stz355. arXiv:1902.00987

W. Baggaley, Radar observations, in Meteors in the Earth's Atmosphere, ed. by E. Murad, I. Williams (Cambridge University Press, Cambridge, 2002), pp. 123-148

M. Benna, D.M. Hurley, T.J. Stubbs, P.R. Mahaffy, R.C. Elphic, Lunar soil hydration constrained by exospheric water liberated by meteoroid impacts. Nat. Geosci. 12, 333-338 (2019)

A.A. Berezhnoy, Chemistry of impact events on Mercury. Icarus 300, 210-222 (2018). https://doi.org/10. 1016/j.icarus.2017.08.034

A.A. Berezhnoy, J. Borovička, Formation of molecules in bright meteors. Icarus 210(1), 150-157 (2010). https://doi.org/10.1016/j.icarus.2010.06.036

A.A. Berezhnoy, K.I. Churyumov, V.V. Kleshchenok, E.A. Kozlova, V. Mangano, Y.V. Pakhomov, V.O. Ponomarenko, V.V. Shevchenko, Y.I. Velikodsky, Properties of the lunar exosphere during the Perseid 2009 meteor shower. Planet. Space Sci. 96, 90-98 (2014). https://doi.org/10.1016/j.pss.2014.03.008. arXiv: 1404.2075

A.A. Berezhnoy, Y.I. Velikodsky, E. Zubko, M. Iten, R. Lena, S. Sposetti, A.A. Tereshchenko, S.I. Popel, E.A. Feoktistova, A.P. Golub', Detection of impact-produced dust clouds near the lunar terminator. Planet. Space Sci. 177, 104689 (2019). https://doi.org/10.1016/j.pss.2019.07.004

O.E. Berg, F.F. Richardson, The Pioneer 8 cosmic dust experiment. Rev. Sci. Instrum. 40, 1333-1337 (1969). https://doi.org/10.1063/1.1683778

T.A. Bida, R.M. Killen, Observations of the minor species al and fe in Mercury's exosphere. Icarus 289, 227-238 (2017). https://doi.org/10.1016/j.icarus.2016.10.019. http://www.sciencedirect.com/science/ article/pii/S0019103516306704

D.L. Bones, J.C. Gómez-Martín, C.J. Empson, J.D. Carrillo-Sánchez, A.D. James, T.P. Conroy, J.M.C. Plane, A novel instrument to measure differential ablation of meteorite samples and proxies: the Meteoric Ablation Simulator (MASI). Rev. Sci. Instrum. 094, 504 (2016). https://doi.org/10.1063/1.4962751.

P. Borin, G. Cremonese, F. Marzari, M. Bruno, S. Marchi, Statistical analysis of micrometeoroids flux on Mercury. Astron. Astrophys. 503(1), 259-264 (2009). https://doi.org/10.1051/0004-6361/200912080

P. Borin, G. Cremonese, F. Marzari, Statistical analysis of the flux of micrometeoroids at Mercury from both cometary and asteroidal components. Astron. Astrophys. 585, A106 (2016a). https://doi.org/10.1051/ 0004-6361/201526767

P. Borin, G. Cremonese, F. Marzari, Statistical analysis of the flux of micrometeoroids at Mercury from both cometary and asteroidal components (Corrigendum). Astron. Astrophys. 588, C3 (2016b). https://doi. org/10.1051/0004-6361/201526767e

P. Borin, G. Cremonese, F. Marzari, A. Lucchetti, Asteroidal and cometary dust flux in the inner solar system. Astron. Astrophys. 605, A94 (2017). https://doi.org/10.1051/0004-6361/201730617

P. Brown, J. Jones, A determination of the strengths of the sporadic radio-meteor sources. Earth Moon Planets 68, 223-245 (1995). https://doi.org/10.1007/BF00671512

P. Brown, R.J. Weryk, D.K. Wong, J. Jones, A meteoroid stream survey using the Canadian Meteor Orbit Radar. I. Methodology and radiant catalogue. Icarus 195, 317-339 (2008). https://doi.org/10.1016/j. icarus.2007.12.002

P. Brown, D.K. Wong, R.J. Weryk, P. Wiegert, A meteoroid stream survey using the Canadian Meteor Orbit Radar. II: Identification of minor showers using a 3D wavelet transform. Icarus 207, 66-81 (2010). https://doi.org/10.1016/j.icarus.2009.11.015

P. Brown, P. Wiegert, D. Clark, E. Tagliaferri, Orbital and physical characteristics of meter-scale impactors from airburst observations. Icarus 266, 96 (2016)

J.S. Bruzzone, D. Janches, P. Jenniskens, R. Weryk, J.L. Hormaechea, A comparative study of radar and optical observations of meteor showers using SAAMER-OS and CAMS. Planet. Space Sci. 188, 104936 (2020). https://doi.org/10.1016/j.pss.2020.104936

M.H. Burger, R.M. Killen, W.E. McClintock, A.W. Merkel, R.J. Vervack, T.A. Cassidy, M. Sarantos, Seasonal variations in Mercury's dayside calcium exosphere. Icarus 238, 51-58 (2014). https://doi.org/10.1016/j. icarus.2014.04.049

S. Cambioni, R. Malhotra, The mid-plane of the Main Asteroid Belt. Astron. J. 155(3), 143 (2018). https:// doi.org/10.3847/1538-3881/aaab6b. arXiv:1801.08096

M.D. Campbell-Brown, High resolution radiant distribution and orbits of sporadic radar meteoroids. Icarus 196, 144-163 (2008). https://doi.org/10.1016/j.icarus.2008.02.022

M. Campbell-Brown, A population of small refractory meteoroids in asteroidal orbits. Planet. Space Sci. 118, 8-13 (2015). https://doi.org/10.1016/j.pss.2015.03.022

M. Campbell-Brown, P. Wiegert, Seasonal variations in the North toroidal sporadic meteor source. Meteorit. Planet. Sci. 44, 1837-1848 (2009). https://doi.org/10.1111/j.1945-5100.2009.tb01992.x 
J.D. Carrillo-Sánchez, J.M.C. Plane, W. Feng, D. Nesvorný, D. Janches, On the size and velocity distribution of cosmic dust particles entering the atmosphere. Geophys. Res. Lett. 42, 6518-6525 (2015). https:// doi.org/10.1002/2015GL065149

J.D. Carrillo-Sánchez, D. Nesvorný, P. Pokorný, D. Janches, J.M.C. Plane, Sources of cosmic dust in the Earth's atmosphere. Geophys. Res. Lett. (2016). https://doi.org/10.1002/2016GL071697

J.D. Carrillo-Sánchez, J.C. Gómez-Martín, D.L. Bones, D. Nesvorný, P. Pokorný, M. Benna, G.J. Flynn, J.M.C. Plane, Cosmic dust fluxes in the atmospheres of Earth, Mars, and Venus. Icarus 335, 113395 (2020). https://doi.org/10.1016/j.icarus.2019.113395

Z. Ceplecha, Influx of interplanetary bodies onto Earth. Astron. Astrophys. 263(1-2), 361-366 (1992)

Z. Ceplecha, J. Borovička, W. Elford, D. Revelle, R. Hawkes, V. Porubčan, M. Šimek, Meteor phenomena and bodies. Space Sci. Rev. 84, 327-471 (1998)

E.L. Christiansen, Performance equations for advanced orbital debris shields, in Space Programs and Technologies Conference (1992), pp. 24-27

A.A. Christou, Predicting martian and venusian meteor shower activity. Earth Moon Planets 95(1-4), 425-431 (2004). https://doi.org/10.1007/s11038-005-9023-0

A.A. Christou, R.M. Killen, M.H. Burger, The meteoroid stream of comet Encke at Mercury: implications for MErcury Surface, Space ENvironment, GEochemistry, and Ranging observations of the exosphere. Geophys. Res. Lett. 42(18), 7311-7318 (2015). https://doi.org/10.1002/2015GL065361

M.J. Cintala, Impact-induced thermal effects in the lunar and Mercurian regoliths. J. Geophys. Res. 97, 947-973 (1992). https://doi.org/10.1029/91JE02207

B.A. Cohen, J.R. Szalay, A.S. Rivkin, J.A. Richardson, R.L. Klima, C.M. Ernst, N.L. Chabot, Z. Sternovsky, M. HoráNyi, Using dust shed from asteroids as microsamples to link remote measurements with meteorite classes. Meteorit. Planet. Sci. 54(9), 2046-2066 (2019). https://doi.org/10.1111/maps. 13348. arXiv: 1906.00876

A. Colaprete, K. Vargo, M. Shirley, D. Land is, D. Wooden, J. Karcz, B. Hermalyn, A. Cook, An overview of the LADEE Ultraviolet-Visible Spectrometer. Space Sci. Rev. 185(1-4), 63-91 (2014). https://doi.org/ 10.1007/s11214-014-0112-0

A. Colaprete, M. Sarantos, D.H. Wooden, T.J. Stubbs, A.M. Cook, M. Shirley, How surface composition and meteoroid impacts mediate sodium and potassium in the lunar exosphere. Science 351(6270), 249-252 (2016)

A. Collette, Z. Sternovsky, M. Horanyi, Production of neutral gas by micrometeoroid impacts. Icarus 227, 89-93 (2014). https://doi.org/10.1016/j.icarus.2013.09.009

G. Cremonese, P. Borin, E. Martellato, F. Marzari, M. Bruno, New calibration of the micrometeoroid flux on Earth. Astrophys. J. Lett. 749(2), L40 (2012). https://doi.org/10.1088/2041-8205/749/2/L40

G. Cremonese, P. Borin, A. Lucchetti, F. Marzari, M. Bruno, Micrometeoroids flux on the Moon. Astron. Astrophys. 551, A27 (2013). https://doi.org/10.1051/0004-6361/201220541

G. Cremonese, F. Capaccioni, M.T. Capria, A. Doressoundiram, P. Palumbo, M. Vincendon, M. Massironi, S. Debei, M. Zusi, F. Altieri, M. Amoroso, G. Aroldi, M. Baroni, A. Barucci, G. Bellucci, J. Benkhoff, S. Besse, C. Bettanini, M. Blecka, D. Borrelli, J.R. Brucato, C. Carli, V. Carlier, P. Cerroni, A. Cicchetti, L. Colangeli, M. Dami, V. Da Deppo, V. Della Corte, M.C. De Sanctis S. Erard, F. Esposito, D. Fantinel, L. Ferranti, F. Ferri, I. FicaiÂ Veltroni, G. Filacchione, E. Flamini, G. Forlani, S. Fornasier, O. Forni, M. Fulchignoni, V. Galluzzi, K. Gwinner, W. Ip, L. Jorda, Y. Langevin, L. Lara, F. Leblanc, C. Leyrat, Y. Li, S. Marchi, L. Marinangeli, F. Marzari, E. MazzottaÂ Epifani, M. Mendillo, V. Mennella, R. Mugnuolo, K. Muinonen, G. Naletto, R. Noschese, E. Palomba, R. Paolinetti, D. Perna, G. Piccioni, R. Politi, F. Poulet, R. Ragazzoni, C. Re, M. Rossi, A. Rotundi, G. Salemi, M. Sgavetti, E. Simioni, N. Thomas, L. Tommasi, A. Turella, T. Van Hoolst, L. Wilson, F. Zambon, A. Aboudan, O. Barraud, N. Bott, P. Borin, G. Colombatti, M. ElÂ Yazidi, S. Ferrari, J. Flahaut, L. Giacomini, L. Guzzetta, A. Lucchetti, E. Martellato, M. Pajola, A. Slemer, G. Tognon, D. Turrini, SIMBIO-SYS: scientific cameras and spectrometer for the BepiColombo mission. Space Sci. Rev. 216(5), 75 (2020). https://doi.org/10. 1007/s11214-020-00704-8

M.M.J. Crismani, N.M. Schneider, J.M.C. Plane, J.S. Evans, S.K. Jain, M.S. Chaffin, J.D. Carrillo-Sanchez, J.I. Deighan, R.V. Yelle, A.I.F. Stewart, W. McClintock, J. Clarke, G.M. Holsclaw, A. Stiepen, F. Montmessin, B.M. Jakosky, Detection of a persistent meteoric metal layer in the Martian atmosphere. Nat. Geosci. 10(6), 401-404 (2017). https://doi.org/10.1038/ngeo2958

M.M.J. Crismani, N.M. Schneider, J.S. Evans, J.M.C. Plane, J.D. Carrillo-Sánchez, S. Jain, J. Deighan, R. Yelle, The impact of comet Siding Spring's meteors on the Martian atmosphere and ionosphere. J. Geophys. Res., Planets 123(10), 2613-2627 (2018). https://doi.org/10.1029/2018JE005750

C.T. D'Aiutolo, W.H. Kinard, R.J. Naumann, Recent NASA meteoroid penetration results from satellites. Smithson. Contrib. Astrophys. 11, 239 (1967)

M. DeLuca, T. Munsat, E. Thomas, Z. Sternovsky, The ionization efficiency of aluminum and iron at meteoric velocities. Planet. Space Sci. 156, 111-116 (2018). https://doi.org/10.1016/j.pss.2017.11.003 
H. Dietzel, G. Eichhorn, H. Fechtig, E. Grun, H.J. Hoffmann, J. Kissel, The HEOS 2 and HELIOS micrometeoroid experiments. J. Phys. E, Sci. Instrum. 6, 209-217 (1973)

V. Dikarev, E. Gruen, J. Baggaley, D. Galligan, M. Landgraf, R. Jehn, The new ESA meteoroid model, in 35th COSPAR Scientific Assembly, vol. 35, ed. by J.P. Paillé (2004), p. 575

F.K. Duennebier, Y. Nakamura, G.V. Latham, H.J. Dorman, Meteoroid storms detected on the Moon. Science 192(4243), 1000-1002 (1976). https://doi.org/10.1126/science.192.4243.1000

G. Eichhorn, Measurements of the light flash produced by high velocity particle impact. Planet. Space Sci. 23, 1519-1525 (1975). https://doi.org/10.1016/0032-0633(75)90005-7

R.C. Elphic, G.T. Delory, B.P. Hine, P.R. Mahaffy, M. Horanyi, A. Colaprete, M. Benna, S.K. Noble, The lunar atmosphere and dust environment explorer mission. Space Sci. Rev. 185, 3-25 (2014). https://doi. org/10.1007/s11214-014-0113-z

J.E. Erickson, Analysis of the meteoroid flux measured by explorer 16 and lunar orbiter. Astron. J. 74, 279 (1969). https://doi.org/10.1086/110807

J.T. Fentzke, D. Janches, A semi-empirical model of the contribution from sporadic meteoroid sources on the meteor input function observed at arecibo. J. Geophys. Res. Space Phys. 113, A03304 (2008). https:// doi.org/10.1029/2007JA012531

J.T. Fentzke, D. Janches, J.J. Sparks, Latitudinal and seasonal variability of the micrometeor input function: a study using model predictions, Arecibo, and PFISR observations. J. Atmos. Sol.-Terr. Phys. 71, 653 (2009)

G.J. Flynn, P. Bleuet, J. Borg, J.P. Bradley, F.E. Brenker, S. Brennan, J. Bridges, D.E. Brownlee, E.S. Bullock, M. Burghammer, B.C. Clark, Z.R. Dai, C.P. Daghlian, Z. Djouadi, S. Fakra, T. Ferroir, C. Floss, I.A. Franchi, Z. Gainsforth, J.P. Gallien, P. Gillet, P.G. Grant, G.A. Graham, S.F. Green, F. Grossemy, P.R. Heck, G.F. Herzog, P. Hoppe, F. Hörz, J. Huth, K. Ignatyev, H.A. Ishii, K. Janssens, D. Joswiak, A.T. Kearsley, H. Khodja, A. Lanzirotti, J. Leitner, L. Lemelle, H. Leroux, K. Luening, G.J. MacPherson, K.K. Marhas, M.A. Marcus, G. Matrajt, T. Nakamura, K. Nakamura-Messenger, T. Nakano, M. Newville, D.A. Papanastassiou, P. Pianetta, W. Rao, C. Riekel, F.J.M. Rietmeijer, D. Rost, C.S. Schwandt, T.H. See, J. Sheffield-Parker, A. Simionovici, I. Sitnitsky, C.J. Snead, F.J. Stadermann, T. Stephan, R.M. Stroud, J. Susini, Y. Suzuki, S.R. Sutton, S. Taylor, N. Teslich, D. Troadec, P. Tsou, A. Tsuchiyama, K. Uesugi, B. Vekemans, E.P. Vicenzi, L. Vincze, J. Westphal Astron, P. Wozniakiewicz, E. Zinner, M.E. Zolensky, Elemental Compositions of Comet 81P/Wild 2 Samples Collected by Stardust. Science 314, 1731 (2006). https://doi.org/10.1126/science.1136141

R. Funase, S. Ikari, K. Miyoshi, Y. Kawabata, S. Nakajima, S. Nomura, N. Funabiki, A. Ishikawa, K. Kakihara, S. Matsushita, R. Takahashi, K. Yanagida, D. Mori, Y. Murata, T. Shibukawa, R. Suzumoto, M. Fujiwara, K. Tomita, H. Aohama, K. Iiyama, S. Ishiwata, H. Kondo, W. Mikuriya, H. Seki, H. Koizumi, J. Asakawa, K. Nishii, A. Hattori, Y. Saito, K. Kikuchi, Y. Kobayashi, A. Tomiki, W. Torii, T. Ito, S. Campagnola, N. Ozaki, N. Baresi, I. Yoshikawa, K. Yoshioka, M. Kuwabara, R. Hikida, S. Arao, S. Abe, M. Yanagisawa, R. Fuse, Y. Masuda, H. Yano, T. Hirai, K. Arai, R. Jitsukawa, E. Ishioka, H. Nakano, T. Ikenaga, T. Hashimoto, Mission to Earth-Moon Lagrange point by a 6U CubeSat: EQUULEUS. IEEE Aerosp. Electron. Syst. Mag. 35(3), 30-44 (2020). https://doi.org/10.1109/MAES.2019.2955577. https://ieeexplore.ieee.org/document/9076200?source=authoralert

C.S. Gardner, A.Z. Liu, D.R. Marsh, W. Feng, J.M.C. Plane, Inferring the global cosmic dust influx to the Earth's atmosphere from lidar observations of the vertical flux of mesospheric Na. J. Geophys. Res. Space Phys. 119(9), 7870-7879 (2014). https://doi.org/10.1002/2014JA020383

J.D. Giorgini, D.K. Yeomans, A.B. Chamberlin, P.W. Chodas, R.A. Jacobson, M.S. Keesey, J.H. Lieske, S.J. Ostro, E.M. Standish, R.N. Wimberly, JPL's on-line solar system data service. Bull. Am. Astron. Soc. 28, 1158 (1996)

J. Gómez Martín, D. Bones, J. Carrillo-Sánchez, J. Trigo-Rodriguez, B. Fegley, J. Plane, Novel experimental simulations of the atmospheric injection of meteoric metals. Astrophys. J. 836, 212 (2017)

J. Grebowsky, J. Moser, W. Pesnell, Meteoric Material - An Important Component of Planetary Atmosphere (Am. Geophys. Union, Washington, 2002), pp. 235-244

J.M. Grebowsky, M. Benna, J.M.C. Plane, G.A. Collinson, P.R. Mahaffy, B.M. Jakosky, Unique, nonEarthlike, meteoritic ion behavior in upper atmosphere of Mars. Geophys. Res. Lett. 44(7), 3066-3072 (2017). https://doi.org/10.1002/2017GL072635

E. Grün, H. Zook, H. Fechtig, R.H. Giese, Collisional balance of the meteoric complex. Icarus 62, 244-272 (1985)

E. Grün, H. Fechtig, M.S. Hanner, J. Kissel, B.A. Lindblad, D. Linkert, D. Maas, G.E. Morfill, H.A. Zook, The Galileo dust detector. Space Sci. Rev. 60, 317-340 (1992)

E. Grün, M. Baguhl, H. Svedhem, H.A. Zook, In situ measurements of cosmic dust, in Astrophys. Space Sci. Library (Springer, Berlin, 2001), p. 295

E. Grün, M. Horanyi, Z. Sternovsky, The lunar dust environment. Planet. Space Sci. 59(14), 1672-1680 (2011). https://doi.org/10.1016/j.pss.2011.04.005 
M. Hajduková, L. Neslusan, The $\chi$-andromedids and January $\alpha$-ursae majorids: a new and a probable shower associated with Comet C/1992 w1 (Ohshita). Icarus, 113960 (2020). https://doi.org/10.1016/j.icarus. 2020.113960. http://www.sciencedirect.com/science/article/pii/S0019103520303316

E.C. Hastings, The explorer XVI micrometeoroid satellite description and preliminary results for the period December 16, 1962 through January 13 1963. Tech. Rep., NASA (1963). https://ntrs.nasa.gov/search. jsp?R=19630002763, tM X-810

G.S. Hawkins, Variation in the occurrence rate of meteors. Astron. J. 61, 386 (1956)

T. Hirai, H. Yano, M. Fujii, S. Hasegawa, N. Moriyama, C. Okamoto, M. Tanaka, Data screening and reduction in interplanetary dust measurement by ikaros-aladdin. Adv. Space Res. 59(6), 1450-1459 (2017). https://doi.org/10.1016/j.asr.2016.12.023. http://www.sciencedirect.com/science/ article/pii/S027311771630727X

M. Horányi, V. Hoxie, D. James, A. Poppe, C. Bryant, B. Grogan, B. Lamprecht, J. Mack, F. Bagenal, S. Batiste, N. Bunch, T. Chanthawanich, F. Christensen, M. Colgan, T. Dunn, G. Drake, A. Fernandez, T. Finley, G. Holland, A. Jenkins, C. Krauss, E. Krauss, O. Krauss, M. Lankton, C. Mitchell, M. Neeland, T. Reese, K. Rash, G. Tate, C. Vaudrin, J. Westfall, The Student Dust Counter on the New Horizons Mission. Space Sci. Rev. 140, 387-402 (2008). https://doi.org/10.1007/s11214-007-9250-y

M. Horányi, Z. Sternovsky, M. Lankton, C. Dumont, S. Gagnard, D. Gathright, E. Grün, D. Hansen, D. James, S. Kempf, B. Lamprecht, R. Srama, J.R. Szalay, G. Wright, The Lunar Dust Experiment (LDEX) onboard the Lunar Atmosphere and Dust Environment Explorer (LADEE) mission. Space Sci. Rev. 185, 93-113 (2014). https://doi.org/10.1007/s11214-014-0118-7

M. Horányi, J.R. Szalay, S. Kempf, J. Schmidt, E. Grün, R. Srama, Z. Sternovsky, A permanent, asymmetric dust cloud around the Moon. Nature 522, 324-326 (2015). https://doi.org/10.1038/nature14479

M. Horányi, E. Bernardoni, S. Kempf, Z. Sternovsky, J. Szalay, Exploration of resources in lunar polar regions, in Lunar and Planetary Science Conference (2020), p. 1465

D.H. Humes, Results of Pioneer 10 and 11 meteoroid experiments - Interplanetary and near-Saturn. J. Geophys. Res. 85, 5841-5852 (1980). https://doi.org/10.1029/JA085iA11p05841

E. Igenbergs, A. Hüdepohl, K. Uesugi, T. Hayashi, H. Svedhem, H. Iglseder, G. Koller, A. Glasmachers, E. Grün, G. Schwehm, H. Mizutani, T. Yamamoto, A. Fujimura, N. Ishii, H. Araki, K. Yamakoshi, K. Nogami, The Munich Dust Counter - a cosmic dust experiment on board of the MUSES-A mission of Japan (1991), Pp 45-48. https://link.springer.com/chapter/10.1007/978-94-011-3640-2_9

D. Janches, C.J. Heinselman, J.L. Chau, A. Chandran, R. Woodman, Modeling the global micrometeor input function in the upper atmosphere observed by high power and large aperture radars. J. Geophys. Res. Space Phys. 111, A07317 (2006). https://doi.org/10.1029/2006JA011628

D. Janches, W. Hocking, S. Pifko, J.L. Hormaechea, D.C. Fritts, C. Brunini, R. Michell, M. Samara, Interferometric meteor head echo observations using the Southern Argentina Agile Meteor Radar. J. Geophys. Res. Space Phys. 119, 2269-2287 (2014). https://doi.org/10.1002/2013JA019241

D. Janches, S. Close, J.L. Hormaechea, N. Swarnalingam, A. Murphy, D. O’Connor, B. Vandepeer, B. Fuller, D.C. Fritts, C. Brunini, The Southern Argentina Agile MEteor Radar Orbital System (SAAMER-OS): an initial sporadic meteoroid orbital survey in the southern sky. Astrophys. J. 809, 36 (2015). https://doi. org/10.1088/0004-637X/809/1/36

D. Janches, N. Swarnalingam, J.D. Carrillo-Sanchez, J.C. Gomez-Martin, R. Marshall, D. Nesvorný, J.M.C. Plane, W. Feng, P. Pokorný, Radar Detectability Studies of Slow and Small Zodiacal Dust Cloud Particles: III. The Role of Sodium and the Head Echo Size on the Probability of Detection. Astrophys. J. 843, 1 (2017). https://doi.org/10.3847/1538-4357/aa775c

D. Janches, P. Pokorný, M. Sarantos, J.R. Szalay, M. Horányi, D. Nesvorný, Constraining the ratio of micrometeoroids from short- and long-period comets at $1 \mathrm{AU}$ from LADEE observations of the lunar dust cloud. Geophys. Res. Lett. 45, 1713-1722 (2018). https://doi.org/10.1002/2017GL076065

D. Janches, C. Brunini, J.L. Hormaechea, A decade of sporadic meteoroid mass distribution indices in the southern hemisphere derived from SAAMER's meteor observations. Astron. J. 157, 240 (2019). https:// doi.org/10.3847/1538-3881/ab1b0f

D. Janches, J.S. Bruzzone, P. Pokorný, J.D. Carrillo-Sanchez, M. Sarantos, A comparative study of the seasonal, temporal, and spatial distribution of meteoroids in the upper atmosphere of Venus, Earth and Mars. Planet. Space Sci. 1, 59 (2020)

D. Janches, J.S. Bruzzone, R.J. Weryk, J.L. Hormaechea, P. Wiegert, C. Brunini, Observations of an unexpected meteor shower outburst at high ecliptic southern latitude and its potential origin. Astrophys. J. Lett. 895(1), L25 (2020b). https://doi.org/10.3847/2041-8213/ab9181

J.M. Jasinski, L.H. Regoli L.H. Cassidy, et al., A transient enhancement of Mercury's exosphere at extremely high altitudes inferred from pickup ions. Nat. Commun. 11, 4350 (2020). https://doi.org/10. 1038/s41467-020-18220-2

P. Jenniskens, Meteor Showers and Their Parent Comets (2006) 
P. Jenniskens, J. Baggaley, I. Crumpton, P. Aldous, P.S. Gural, D. Samuels, J. Albers, R. Soja, A surprise southern hemisphere meteor shower on New-Year's Eve 2015: the Volantids (IAU\#758, VOL). J. Int. Meteor Organ. 44, 35-41 (2016)

C.L. Johnson, S.A. Hauck II, A whole new Mercury: messenger reveals a dynamic planet at the last frontier of the inner solar system. J. Geophys. Res., Planets 121(11), 2349-2362 (2016). https://doi.org/10.1002/ 2016JE005150. https://agupubs.onlinelibrary.wiley.com/doi/pdf/10.1002/2016JE005150

J. Jones, P. Brown, Sporadic meteor radiant distribution: orbital survey results. Mon. Not. R. Astron. Soc. 265, 524-532 (1993)

M.S.P. Kelley, T.L. Farnham, D. Bodewits, P. Tricarico, D. Farnocchia, A study of dust and gas at Mars from Comet C/2013 A1 (Siding Spring). Astrophys. J. Lett. 792(1), L16 (2014). https://doi.org/10.1088/20418205/792/1/L16. arXiv:1408.2792

J. Kero, M.D. Campbell-Brown, G. Stober, J.L. Chau, J.D. Mathews, A. Pellinen-Wannberg, Radar Observations of Meteors. (2019), p. 65

D. Kessler, Average relative velocity of sporadic meteoroids in interplanetary space. AIAA J. 7(12), 2337-2338 (1969). https://doi.org/10.2514/3.5539

D.J. Kessler, Derivation of the collision probability between orbiting objects the lifetimes of Jupiter's outer moons. Icarus 48, 39-48 (1981). https://doi.org/10.1016/0019-1035(81)90151-2

R.M. Killen, J.M. Hahn, Impact vaporization as a possible source of Mercury's calcium exosphere. Icarus 250, 230-237 (2015). https://doi.org/10.1016/j.icarus.2014.11.035

E.D. Kondrateva, E.A. Reznikov, Comet Tempel-Tuttle and the Leonid meteor swarm. Astron. Vestn. 19, 144-151 (1985)

D. Koschny, E. Grün, Impacts into ice-silicate mixtures: ejecta mass and size distributions. Icarus 154, 402-411 (2001). https://doi.org/10.1006/icar.2001.6708

P. Koten, J. Rendtel, L. Shrbený, P. Gural, J. Borovička, P. Kozak, Meteors and meteor showers as observed by optical techniques, in Meteoroids: Sources of Meteors on Earth and Beyond, ed. by G.O. Ryabova, D.J. Asher, M.D. Campbell-Brown (Cambridge University Press, Cambridge, 2019), pp. 90-115. ISBN 9781108426718

H. Krüger, A.V. Krivov, M. Sremčević, E. Grün, Impact-generated dust clouds surrounding the Galilean moons. Icarus 164(1), 170-187 (2003). https://doi.org/10.1016/S0019-1035(03)00127-1

H. Krüger, P. Strub, R. Srama, M. Kobayashi, T. Arai, H. Kimura, T. Hirai, G. Moragas-Klostermeyer, N. Altobelli, V.J. Sterken, J. Agarwal, M. Sommer, E. Grün, Modelling DESTINY ${ }^{+}$interplanetary and interstellar dust measurements en route to the active asteroid (3200) Phaethon. Planet. Space Sci. 172, 22-42 (2019). https://doi.org/10.1016/j.pss.2019.04.005. arXiv:1904.07384

C. Leinert, M. Hanner, I. Richter, E. Pitz, The plane of symmetry of interplanetary dust in the inner solar system. Astron. Astrophys. 82, 328-336 (1980)

H.F. Levison, M.J. Duncan, From the Kuiper belt to Jupiter-family comets: the spatial distribution of ecliptic comets. Icarus 127, 13-32 (1997). https://doi.org/10.1006/icar.1996.5637

H.F. Levison, M.J. Duncan, L. Dones, B.J. Gladman, The scattered disk as a source of Halley-type comets. Icarus 184, 619-633 (2006). https://doi.org/10.1016/j.icarus.2006.05.008

A. Liakos, A.Z. Bonanos, E.M. Xilouris, D. Koschny, I. Bellas-Velidis, P. Boumis, V. Charmandaris, A. Dapergolas, A. Fytsilis, A. Maroussis, R. Moissl, NELIOTA: methods, statistics, and results for meteoroids impacting the Moon. Astron. Astrophys. 633, A112 (2020). https://doi.org/10.1051/0004-6361/ 201936709. arXiv:1911.06101

S.G. Love, J.H. Allton, Micrometeoroid impact crater statistics at the boundary of Earth's gravitational sphere of influence. Icarus 184, 302-307 (2006). https://doi.org/10.1016/j.icarus.2006.05.023

S. Love, D.E. Brownlee, A direct measurement of the terrestrial mass accretion rate of cosmic dust. Science 262, 550-553 (1993)

E.J. Lyytinen, T. Van Flandern, Predicting the strength of Leonid outbursts. Earth Moon Planets 82, 149-166 (2000)

J.M. Madiedo, J.L. Ortiz, M. Yanagisawa, J. Aceituno, F. Aceituno, Impact flashes of meteoroids on the Moon, in Meteoroids: Sources of Meteors on Earth and Beyond, ed. by G.O. Ryabova, D.J. Asher, M.D. Campbell-Brown (Cambridge University Press, Cambridge, 2019), pp. 136-158. ISBN 9781108426718

P.R. Mahaffy, R. Richard Hodges, M. Benna, T. King, R. Arvey, M. Barciniak, M. Bendt, D. Carigan, T. Errigo, D.N. Harpold, V. Holmes, C.S. Johnson, J. Kellogg, P. Kimvilakani, M. Lefavor, J. Hengemihle, F. Jaeger, E. Lyness, J. Maurer, D. Nguyen, T.J. Nolan, F. Noreiga, M. Noriega, K. Patel, B. Prats, O. Quinones, E. Raaen, F. Tan, E. Weidner, M. Woronowicz, C. Gundersen, S. Battel, B.P. Block, K. Arnett, R. Miller, C. Cooper, C. Edmonson, The Neutral Mass Spectrometer on the Lunar Atmosphere and Dust Environment Explorer Mission. Space Sci. Rev. 185(1-4), 27-61 (2014). https://doi.org/10. 1007/s11214-014-0043-9

V. Mangano, A. Milillo, A. Mura, S. Orsini, E. De Angelis, P. Di Lellis, A.M. Wurz, The contribution of impulsive meteoritic impact vapourization to the Hermean exosphere. Planet. Space Sci. 55(11), 1541-1556 (2007). https://doi.org/10.1016/j.pss.2006.10.008 
I. Mann, L. Nouzák, J. Vaverka, T. Antonsen, Å. Fredriksen, K. Issautier, D. Malaspina, N. Meyer-Vernet, J. Pavlů, Z. Sternovsky, J. Stude, S. Ye, A. Zaslavsky, Dust observations with antenna measurements and its prospects for observations with parker solar probe and solar orbiter. Ann. Geophys. 37(6), 1121-1140 (2019). https://doi.org/10.5194/angeo-37-1121-2019. https://www.ann-geophys.net/37/1121/2019/

S. Marchi, A. Morbidelli, G. Cremonese, Flux of meteoroid impacts on Mercury. Astron. Astrophys. 431(3), 1123-1127 (2005). https://doi.org/10.1051/0004-6361:20041800

D. Marsh, D. Janches, W. Feng, J. Plane, A global model of meteoric sodium. J. Geophys. Res., Atmos. 118, 11,442-11,452 (2013). https://doi.org/10.1002/jgrd.50870

J.D. Mathews, D. Janches, D. Meisel, Q. Zhou, The micrometeoroid mass flux into the upper atmosphere: Arecibo results and a comparison with prior estimates. Geophys. Res. Lett. 28(10), 1929-1932 (2001)

W.E. McClintock, R.J. Vervack, E.T. Bradley, R.M. Killen, N. Mouawad, A.L. Sprague, M.H. Burger, S.C. Solomon, N.R. Izenberg, Messenger observations of Mercury's exosphere: detection of magnesium and distribution of constituents. Science 324(5927), 610-613 (2009). https://doi.org/10.1126/science. 1172525. https://science.sciencemag.org/content/324/5927/610.full.pdf

D.J. McComas, E.R. Christian, N.A. Schwadron, N. Fox, J. Westlake, F. Allegrini, D.N. Baker, D. Biesecker, M. Bzowski, G. Clark, C.M.S. Cohen, I. Cohen, M.A. Dayeh, R. Decker, G.A. de Nolfo, M.I. Desai, R.W. Ebert, H.A. Elliott, H. Fahr, P.C. Frisch, H.O. Funsten, S.A. Fuselier, A. Galli, A.B. Galvin, J. Giacalone, M. Gkioulidou, F. Guo, M. Horanyi, P. Isenberg, P. Janzen, L.M. Kistler, K. Korreck, M.A. Kubiak, H. Kucharek, B.A. Larsen, R.A. Leske, N. Lugaz, J. Luhmann, W. Matthaeus, D. Mitchell, E. Moebius, K. Ogasawara, D.B. Reisenfeld, J.D. Richardson, C.T. Russell, J.M. Sokół, H.E. Spence, R. Skoug, Z. Sternovsky, P. Swaczyna, J.R. Szalay, M. Tokumaru, M.E. Wiedenbeck, P. Wurz, G.P. Zank, E.J. Zirnstein, Interstellar Mapping and Acceleration Probe (IMAP): a new NASA mission. Space Sci. Rev. 214(8), 116 (2018). https://doi.org/10.1007/s11214-018-0550-1

R.H. McNaught, D.J. Asher, Leonid dust trails and meteor storms. J. Int. Meteor Organ. 27(2), 85-102 (1999)

A.W. Merkel, T.A. Cassidy, R.J. Vervack, W.E. McClintock, M. Sarantos, M.H. Burger, R.M. Killen, Seasonal variations of Mercury's magnesium dayside exosphere from MESSENGER observations. Icarus 281, 46-54 (2017). https://doi.org/10.1016/j.icarus.2016.08.032

J. Miao, J.P.W. Stark, Direct simulation of meteoroids and space debris flux on LDEF spacecraft surfaces. Planet. Space Sci. 49, 927-935 (2001). https://doi.org/10.1016/S0032-0633(01)00042-3

A.V. Moorhead, A. Kingery, S. Ehlert, NASA's Meteoroid Engineering Model 3 and its ability to replicate spacecraft impact rates. J. Spacecr. Rockets 57, 160-176 (2020). https://doi.org/10.2514/1.A34561. arXiv:1909.05947

A. Morbidelli, B. Gladman, Orbital and temporal distributions of meteorites originating in the asteroid belt. Meteorit. Planet. Sci. 33(5), 999-1016 (1998). https://doi.org/10.1111/j.1945-5100.1998.tb01707.x

D. Nesvorný, D. Vokrouhlický, W.F. Bottke, M. Sykes, Physical properties of asteroid dust bands and their sources. Icarus 181, 107-144 (2006). https://doi.org/10.1016/j.icarus.2005.10.022

D. Nesvorný, P. Jenniskens, H.F. Levison, W.F. Bottke, D. Vokrouhlický, M. Gounelle, Cometary origin of the zodiacal cloud and carbonaceous micrometeorites. Implications for hot debris disks. Astrophys. J. 713, 816-836 (2010). https://doi.org/10.1088/0004-637X/713/2/816. arXiv:0909.4322

D. Nesvorný, D. Janches, D. Vokrouhlický, P. Pokorný, W.F. Bottke, P. Jenniskens, Dynamical model for the zodiacal cloud and sporadic meteors. Astrophys. J. 743, 129 (2011a). https://doi.org/10.1088/0004637X/743/2/129. arXiv:1109.2983

D. Nesvorný, D. Vokrouhlický, P. Pokorný, D. Janches, Dynamics of dust particles released from Oort Cloud Comets and their contribution to radar meteors. Astrophys. J. 743, 37 (2011b). https://doi.org/10.1088/ 0004-637X/743/1/37. arXiv:1109.2981

K. Nogami, M. Fujii, H. Ohashi, T. Miyachi, S. Sasaki, S. Hasegawa, H. Yano, H. Shibata, T. Iwai, S. Minami, S. Takechi, E. Grün, R. Srama, Development of the Mercury Dust Monitor (MDM) onboard the BepiColombo mission. Planet. Space Sci. 58(1-2), 108-115 (2010). https://doi.org/10.1016/j.pss.2008. 08.016

J. Oberst, Y. Nakamura, A search for clustering among the meteoroid impacts detected by the Apollo lunar seismic network. Icarus 91(2), 315-325 (1991). https://doi.org/10.1016/0019-1035(91)90027-Q

R. O'Neal, L.R. Center, U.S.N. Aeronautics, S. Administration, The Explorer XXIII micrometeoroid satellite description and preliminary results for the period November 6, 1964 through February 151965 . NASA technical memorandum, National Aeronautics and Space Administration (1965). https://books.google. co.jp/books?id=aVOF141pwvIC

E. Pierazzo, N. Artemieva, E. Asphaug, E.C. Baldwin, J. Cazamias, R. Coker, G.S. Collins, D.A. Crawford, T. Davison, D. Elbeshausen, K.A. Holsapple, K.R. Housen, D.G. Korycansky, K. Wünnemann, Validation of numerical codes for impact and explosion cratering: impacts on strengthless and metal targets. Meteorit. Planet. Sci. 43(12), 1917-1938 (2008). https://doi.org/10.1111/j.1945-5100.2008.tb00653.x

C.M. Pieters, S.K. Noble, Space weathering on airless bodies. J. Geophys. Res., Planets 121, 1865-1884 (2016). https://doi.org/10.1002/2016JE005128 
S. Pifko, D. Janches, S. Close, J. Sparks, T. Nakamura, D. Nesvorny, The meteoroid input function and predictions of mid-latitude meteor observations by the MU radar. Icarus 223, 444-459 (2013). https:// doi.org/10.1016/j.icarus.2012.12.014

C. Plainaki, A. Mura, A. Milillo, S. Orsini, S. Livi, V. Mangano, S. Massetti, R. Rispoli, E. De Angelis, Investigation of the possible effects of comet Encke's meteoroid stream on the Ca exosphere of Mercury. J. Geophys. Res., Planets 122(6), 1217-1226 (2017). https://doi.org/10.1002/2017JE005304

J. Plane, Atmospheric chemistry of meteoric metals. Chem. Rev. 103(12), 4963-4984 (2003)

J.M.C. Plane, Cosmic dust in the Earth's atmosphere. Chem. Soc. Rev. 41, 6507-6518, 2012 41:6507-6518 (2012). https://doi.org/10.1039/c2cs35132c

J. Plane, W. Feng, E. Dawkins, The mesosphere and metals: chemistry and changes. Chem. Rev. 115, 4497-4541 (2015). https://doi.org/10.1021/cr500501m

J.M.C. Plane, G.J. Flynn, A. Määttänen, J.E. Moores, A.R. Poppe, J.D. Carrillo-Sánchez, C. Listowski, Impacts of cosmic dust on planetary atmospheres and surfaces. Space Sci. Rev. 214(1), 23 (2018). https:// doi.org/10.1007/s11214-017-0458-1

P. Pokorný, P.G. Brown, A reproducible method to determine the meteoroid mass index. Astron. Astrophys. 592, A150 (2016). https://doi.org/10.1051/0004-6361/201628134. arXiv:1605.04437

P. Pokorný, D. Vokrouhlický, D. Nesvorný, M. Campbell-Brown, P. Brown, Dynamical model for the toroidal sporadic meteors. Astrophys. J. 789, 25 (2014). https://doi.org/10.1088/0004-637X/789/1/25

P. Pokorný, D. Janches, P.G. Brown, J.L. Hormaechea, An orbital meteoroid stream survey using the Southern Argentina Agile MEteor Radar (SAAMER) based on a wavelet approach. Icarus 290, 162-182 (2017a). https://doi.org/10.1016/j.icarus.2017.02.025

P. Pokorný, M. Sarantos, D. Janches, Reconciling the dawn-dusk asymmetry in Mercury's exosphere with the micrometeoroid impact directionality. Astrophys. J. Lett. 842, L17 (2017b). https://doi.org/10.3847/ 2041-8213/aa775d. arXiv:1706.01461

P. Pokorný, M. Sarantos, D. Janches, A comprehensive model of the meteoroid environment around Mercury. Astrophys. J. 863, 31 (2018). https://doi.org/10.3847/1538-4357/aad051. arXiv:1807.02749

P. Pokorný, D. Janches, M. Sarantos, J.R. Szalay, M. Horányi, D. Nesvorný, M.J. Kuchner, Meteoroids at the Moon: orbital properties, surface vaporization, and impact ejecta production. J. Geophys. Res., Planets 124, 752-778 (2019). https://doi.org/10.1029/2018JE005912

P. Pokorný, M. Sarantos, D. Janches, E. Mazarico, Meteoroid bombardment of lunar poles. Astrophys. J. 894(2), 114 (2020). https://doi.org/10.3847/1538-4357/ab83ee. arXiv:2003.12640

A.R. Poppe, An improved model for interplanetary dust fluxes in the outer Solar System. Icarus 264, 369-386 (2016). https://doi.org/10.1016/j.icarus.2015.10.001

A. Poppe, D. James, M. Horányi, Measurements of the terrestrial dust influx variability by the Cosmic Dust Experiment. Planet. Space Sci. 59, 319-326 (2011). https://doi.org/10.1016/j.pss.2010.12.002

V. Porubčan, L. Kornoš, I.P. Williams, The Taurid complex meteor showers and asteroids. Contrib. Astron. Obs. Skaln. Pleso 36(2), 103-117 (2006). arXiv:0905.1639

F. Postberg, E. Grün, M. Horanyi, S. Kempf, H. Krüger, J. Schmidt, F. Spahn, R. Srama, Z. Sternovsky, M. Trieloff, Compositional mapping of planetary moons by mass spectrometry of dust ejecta. Planet. Space Sci. 59(14), 1815-1825 (2011). https://doi.org/10.1016/j.pss.2011.05.001

E. Quémerais, J.Y. Chaufray, D. Koutroumpa, F. Leblanc, A. Reberac, B. Lustrement, C. Montaron, J.F. Mariscal, N. Rouanet, I. Yoshikawa, G. Murakami, K. Yoshioka, O. Korablev, D. Belyaev, M.G. Pelizzo, A. Corso, P. Zuppella, PHEBUS on Bepi-Colombo: post-launch update and instrument performance. Space Sci. Rev. 216(4), 67 (2020). https://doi.org/10.1007/s11214-020-00695-6

J.W. Rhee, O.E. Berg, F.F. Richardson, Heliocentric distribution of cosmic dust intercepted by Pioneer 8 and 9. Geophys. Res. Lett. 1(8), 345-346 (1974). https://doi.org/10.1029/GL001i008p00345

M. Rowan-Robinson, B. May, An improved model for the infrared emission from the zodiacal dust cloud: cometary, asteroidal and interstellar dust. Mon. Not. R. Astron. Soc. 429(4), 2894-2902 (2013). https:// doi.org/10.1093/mnras/sts471. arXiv:1212.4759

J.M. Russell, S.M. Bailey, L.L. Gordley, D.W. Rusch, M. Horányi, M.E. Hervig, G.E. Thomas, C.E. Randall, D.E. Siskind, M.H. Stevens, M.E. Summers, M.J. Taylor, C.R. Englert, P.J. Espy, W.E. McClintock, A.W. Merkel, The Aeronomy of Ice in the Mesosphere (AIM) mission: overview and early science results. J. Atmos. Sol.-Terr. Phys. 71, 289-299 (2009). https://doi.org/10.1016/j.jastp.2008.08.011

M. Sarantos, R.M. Killen, A. Surjalal Sharma, J.A. Slavin, Sources of sodium in the lunar exosphere: modeling using ground-based observations of sodium emission and spacecraft data of the plasma. Icarus $\mathbf{2 0 5}$, 364-374 (2010). https://doi.org/10.1016/j.icarus.2009.07.039

M. Sarantos, R.M. Killen, W.E. McClintock, E.T. Bradley, R.J. Vervack, M. Benna, J.A. Slavin, Limits to Mercury's magnesium exosphere from MESSENGER second flyby observations. Planet. Space Sci. 59(15), 1992-2003 (2011)

M. Sarantos, R.E. Hartle, R.M. Killen, Y. Saito, J.A. Slavin, A. Glocer, Flux estimates of ions from the lunar exosphere. Geophys. Res. Lett. 39, L13101 (2012a). https://doi.org/10.1029/2012GL052001 
M. Sarantos, R.M. Killen, D.A. Glenar, M. Benna, T.J. Stubbs, Metallic species, oxygen and silicon in the lunar exosphere: upper limits and prospects for LADEE measurements. J. Geophys. Res. 117, A03103 (2012b)

B.V. Sarli, M. Horikawa, C.H. Yam, Y. Kawakatsu, T. Yamamoto, DESTINY ${ }^{+}$trajectory design to (3200) Phaethon. J. Astronaut. Sci. 65(1), 82-110 (2018)

S. Sasaki, E. Igenbergs, H. Ohashi, R. Senger, R. Münzenmayer, W. Naumann, E. Grün, K. Nogami, I. Mann, H. Svedhem, Summary of interplanetary and interstellar dust observation by Mars Dust Counter on board NOZOMI. Adv. Space Res. 39(3), 485-488 (2007). https://doi.org/10.1016/j.asr.2006.11.006

N.M. Schneider, J.I. Deighan, A.I.F. Stewart, W.E. McClintock, S.K. Jain, M.S. Chaffin, A. Stiepen, M. Crismani, J.M.C. Plane, J.D. Carrillo-Sánchez, J.S. Evans, M.H. Stevens, R.V. Yelle, J.T. Clarke, G.M. Holsclaw, F. Montmessin, B.M. Jakosky, MAVEN IUVS observations of the aftermath of the Comet Siding Spring meteor shower on Mars. Geophys. Res. Lett. 42(12), 4755-4761 (2015). https://doi.org/ 10.1002/2015GL063863

C. Schult, G. Stober, D. Janches, J.L. Chau, Results of the first continuous meteor head echo survey at polar latitudes. Icarus 297, 1-13 (2017). https://doi.org/10.1016/j.icarus.2017.06.019

Z. Sekanina, Statistical model of meteor streams. IV - a study of radio streams from the synoptic year. Icarus 27, 265-321 (1976)

F. Selsis, J. Brillet, M. Rapaport, Meteor showers of cometary origin in the Solar System: revised predictions. Astron. Astrophys. 416, 783-789 (2004). https://doi.org/10.1051/0004-6361:20031724

S.M. Smith, J.K. Wilson, J. Baumgardner, M. Mendillo, Discovery of the distant lunar sodium tail and its enhancement following the Leonid Meteor Shower of 1998. Geophys. Res. Lett. 26(12), 1649-1652 (1999). https://doi.org/10.1029/1999GL900314

R. Srama, T.J. Ahrens, N. Altobelli, S. Auer, J.G. Bradley, M. Burton, V.V. Dikarev, T. Economou, H. Fechtig, M. Görlich, M. Grande, A. Graps, E. Grün, O. Havnes, S. Helfert, M. Horányi, E. Igenbergs, E.K. Jessberger, T.V. Johnson, S. Kempf, A.V. Krivov, H. Krüger, A. Mocker-Ahlreep, G. Moragas-Klostermeyer, P. Lamy, M. Landgraf, D. Linkert, G. Linkert, F. Lura, J.A.M. McDonnell, D. Möhlmann, G.E. Morfill, M. Müller, M. Roy, G. Schäfer, G. Schlotzhauer, G.H. Schwehm, F. Spahn, M. Stübig, J. Svestka, V. Tschernjawski, J. Tuzzolino Astron, R. Wäsch, H.A. Zook, The Cassini cosmic dust analyzer. Space Sci. Rev. 114, 465-518 (2004). https://doi.org/10.1007/s11214-004-1435-Z

C.C. Stark, M.J. Kuchner, A new algorithm for self-consistent three-dimensional modeling of collisions in dusty debris disks. Astrophys. J. 707(1), 543-553 (2009). https://doi.org/10.1088/0004-637X/707/1/ 543. arXiv:0909.2227

D.I. Steel, W.G. Elford, Collisions in the solar system. III-meteoroid survival times. Mon. Not. R. Astron. Soc. 218, 185-199 (1986). https://doi.org/10.1093/mnras/218.2.185

G. Stenborg, J.R. Stauffer, R.A. Howard, Evidence for a circumsolar dust ring near Mercury's orbit. Astrophys. J. 868(1), 74 (2018). https://doi.org/10.3847/1538-4357/aae6cb

S.A. Stern, The lunar atmosphere: history, status, current problems, and context. Rev. Geophys. 37(4), 453-492 (1999). https://doi.org/10.1029/1999RG900005

R.M. Suggs, D.E. Moser, W.J. Cooke, R.J. Suggs, The flux of kilogram-sized meteoroids from lunar impact monitoring. Icarus 238, 23 (2014)

S. Sugita, P.H. Schultz, M.A. Adams, Spectroscopic measurements of vapor clouds due to oblique impacts. J. Geophys. Res. 103(E8), 19,427-19,441 (1998). https://doi.org/10.1029/98JE02026.

S. Sugita, P.H. Schultz, S. Hasegawa, Intensities of atomic lines and molecular bands observed in impactinduced luminescence. J. Geophys. Res., Planets 108(E12), 5140 (2003). https://doi.org/10.1029/ 2003JE002156

N. Swarnalingam, D. Janches, J.D. Carrillo-Sanchez, P. Pokorny, J.M.C. Plane, Z. Sternovsky, D. Nesvorny, Modeling the altitude distribution of meteor head echoes observed with HPLA radars: implications for the radar detectability of meteoroid populations. Astron. J. 157, 179 (2019). https://doi.org/10.3847/ 1538-3881/ab0ec6

J.R. Szalay, M. Horányi, Annual variation and synodic modulation of the sporadic meteoroid flux to the Moon. Geophys. Res. Lett. 42, 10 (2015). https://doi.org/10.1002/2015GL066908

J.R. Szalay, M. Horányi, The search for electrostatically lofted grains above the Moon with the Lunar Dust Experiment. Geophys. Res. Lett. 42(13), 5141-5146 (2015b). https://doi.org/10.1002/2015GL064324

J.R. Szalay, M. Horányi, Detecting meteoroid streams with an in-situ dust detector above an airless body. Icarus 275, 221-231 (2016). https://doi.org/10.1016/j.icarus.2016.04.024

J.R. Szalay, M. Horányi, The impact ejecta environment of near Earth asteroids. Astrophys. J. Lett. 830(2), L29 (2016b). https://doi.org/10.3847/2041-8205/830/2/L29. http://stacks.iop.org/2041-8205/830/i=2/ $\mathrm{a}=\mathrm{L} 29$

J.R. Szalay, M. Horányi, Lunar meteoritic gardening rate derived from in situ LADEE/LDEX measurements. Geophys. Res. Lett. 43(10), 4893-4898 (2016c). https://doi.org/10.1002/2016GL069148 
J.R. Szalay, M. Horányi, A. Colaprete, M. Sarantos, Meteoritic influence on sodium and potassium abundance in the lunar exosphere measured by LADEE. Geophys. Res. Lett. 43(12), 6096-6102 (2016). https://doi. org/10.1002/2016GL069541

J.R. Szalay, P. Pokorný, P. Jenniskens, M. Horányi, Activity of the 2013 Geminid meteoroid stream at the Moon. Mon. Not. R. Astron. Soc. 474(3), 4225-4231 (2018). https://doi.org/10.1093/mnras/stx3007

J.R. Szalay, P. Pokorný, M. Horányi, D. Janches, M. Sarantos, R. Srama, Impact ejecta environment of an eccentric asteroid: 3200 Phaethon. Planet. Space Sci. 165, 194-204 (2019). https://doi.org/10.1016/j. pss.2018.11.001

J.R. Szalay, P. Pokorný, S.D. Bale, E.R. Christian, K. Goetz, K. Goodrich, M.E. Hill, M. Kuchner, R. Larsen, D. Malaspina, D.J. McComas, D. Mitchell, B. Page, N. Schwadron, The near-Sun dust environment: initial observations from Parker Solar Probe. Astrophys. J. Suppl. Ser. 246(2), 27 (2020a). https://doi. org/10.3847/1538-4365/ab50c1. arXiv:1912.02639

J.R. Szalay, P. Pokorný, M. Horányi, Hyperbolic meteoroids impacting the Moon. Astrophys. J. Lett. 890(1), L11 (2020b). https://doi.org/10.3847/2041-8213/ab7195

M. Tabata, H. Kawai, H. Yano, E. Imai, H. Hashimoto, S. Yokobori A. Yamagishi, Ultralow-density doublelayer silica aerogel fabrication for the intact capture of cosmic dust in low-Earth orbits. J. Sol-Gel Sci. Technol. 77(2), 325-334 (2016). https://doi.org/10.1007/s10971-015-3857-3

A. Taylor, The Harvard radio meteor project meteor velocity distribution reappraised. Icarus 116, 205-209 (1995)

S. Taylor, J.H. Lever, R.P. Harvey, Accretion rate of cosmic spherules measured at the South Pole. Nature 392(6679), 899-903 (1998). https://doi.org/10.1038/31894

E. Thomas, M. Horányi, D. Janches, T. Munsat, J. Simolka, Z. Sternovsky, Measurements of the ionization coefficient of simulated iron micrometeoroids. Geophys. Res. Lett. 43, 3645-3652 (2016). https://doi. org/10.1002/2016GL068854

J.I. Thorpe, J. Slutsky, J.G. Baker, T.B. Littenberg, S. Hourihane, N. Pagane, P. Pokorny, D. Janches, M. Armano, H. Audley, G. Auger, J. Baird, M. Bassan, P. Binetruy, M. Born, D. Bortoluzzi, N. Brandt, M. Caleno, A. Cavalleri, A. Cesarini, A.M. Cruise, K. Danzmann, M. de Deus Silva, R. De Rosa, L. Di Fiore, I. Diepholz, G. Dixon, R. Dolesi, N. Dunbar, L. Ferraioli, V. Ferroni, E.D. Fitzsimons, R. Flatscher, M. Freschi, C. García Marirrodriga, R. Gerndt, L. Gesa, F. Gibert, D. Giardini, R. Giusteri, A. Grado, C. Grimani, J. Grzymisch, I. Harrison, G. Heinzel, M. Hewitson, D. Hollington, D. Hoyland, M. Hueller, H. Inchauspé, O. Jennrich, P. Jetzer, B. Johlander, N. Karnesis, B. Kaune, N. Korsakova, C.J. Killow, J.A. Lobo, I. Lloro, L. Liu, J.P. López-Zaragoza, R. Maarschalkerweerd, D. Mance, V. Martín, L. Martin-Polo, J. Martino, F. Martin-Porqueras, S. Madden, I. Mateos, P.W. McNamara, J. Mendes, L. Mendes, M. Nofrarias, S. Paczkowski, M. Perreur-Lloyd, A. Petiteau, P. Pivato, E. Plagnol, P. Prat, U. Ragnit, J. Ramos-Castro, J. Reiche, D.I. Robertson, H. Rozemeijer, F. Rivas, G. Russano, P. Sarra, A. Schleicher, D. Shaul, C.F. Sopuerta, R. Stanga, T. Sumner, D. Texier, C. Trenkel, M. Tröbs, D. Vetrugno, S. Vitale, G. Wanner, H. Ward, P. Wass, D. Wealthy, W.J. Weber, L. Wissel, A. Wittchen, A. Zambotti, C. Zanoni, T. Ziegler, P. Zweifel, C. Cutler, N. Demmons, C. Dunn, M. Girard, O. Hsu, S. Javidnia, I. Li, P. Maghami, C. Marrese-Reading, J. Mehta, J. O’Donnell, A. Romero-Wolf, J. Ziemer (LISA Pathfinder Collaboration P ST7-DRS Operations Team, Barela), Micrometeoroid events in LISA Pathfinder. Astrophys. J. 883(1), 53 (2019). https://doi.org/10.3847/1538-4357/ab3649. arXiv:1905.02765

J. Vaubaillon, F. Colas, L. Jorda, A new method to predict meteor showers. II. Application to the Leonids. Astron. Astrophys. 439(2), 761-770 (2005). https://doi.org/10.1051/0004-6361:20042626

J. Vaubaillon, L. Maquet, R. Soja, Meteor hurricane at Mars on 2014 October 19 from comet C/2013 A1. Mon. Not. R. Astron. Soc. 439(4), 3294-3299 (2014). https://doi.org/10.1093/mnras/stu160

S. Verani, C. Barbieri, C. Benn, G. Cremonese, Possible detection of meteor stream effects on the lunar sodium atmosphere. Planet. Space Sci. 46(8), 1003-1006 (1998). https://doi.org/10.1016/S00320633(98)00024-5

S. Verani, C. Barbieri, C.R. Benn, G. Cremonese, M. Mendillo, The 1999 Quadrantids and the lunar Na atmosphere. Mon. Not. R. Astron. Soc. 327(1), 244-248 (2001). https://doi.org/10.1046/j.1365-8711. 2001.04748.x. arXiv:astro-ph/0106447

R.J. Vervack, W.E. McClintock, R.M. Killen, A.L. Sprague, B.J. Anderson, M.H. Burger, E.T. Bradley, N. Mouawad, S.C. Solomon, N.R. Izenberg, Mercury's complex exosphere: results from MESSENGER's third flyby. Science 329(5992), 672 (2010). https://doi.org/10.1126/science. 1188572

R.J. Vervack, R.M. Killen, W.E. McClintock, A.W. Merkel, M.H. Burger, T.A. Cassidy, M. Sarantos, New discoveries from MESSENGER and insights into Mercury's exosphere. Geophys. Res. Lett. 43(22), 11,545-11,551 (2016). https://doi.org/10.1002/2016GL071284

A.A. Weiss, J.W. Smith, A southern hemisphere survey of the radiants of sporadic meteors. Mon. Not. R. Astron. Soc. 121, 5 (1960)

F.L. Whipple, Photographic meteor studies. III. The Taurid shower. Proc. Am. Philos. Soc. 83, 711-745 (1940) 
P. Wiegert, J. Vaubaillon, M. Campbell-Brown, A dynamical model of the sporadic meteoroid complex. Icarus 201, 295-310 (2009). https://doi.org/10.1016/j.icarus.2008.12.030

P. Wiegert, P. Brown, P. Pokorný, Q. Ye, C. Craig, K. Lenartowicz, Z. Krzeminski, D. Clark, Supercatastrophic disruption of asteroids in the context of SOHO comet, fireball, and meteor observations. Astron. J. 159(4), 143 (2020). https://doi.org/10.3847/1538-3881/ab700d

Publisher's Note Springer Nature remains neutral with regard to jurisdictional claims in published maps and institutional affiliations.

\title{
Authors and Affiliations
}

\section{Diego Janches ${ }^{1}$ - Alexey A. Berezhnoy ${ }^{2,3}$ - Apostolos A. Christou ${ }^{4}$. Gabriele Cremonese ${ }^{5}$. Takayuki Hirai ${ }^{6}$. Mihály Horányi ${ }^{7,8,9}$. Jamie M. Jasinski ${ }^{10}$. Menelaos Sarantos ${ }^{11}$}

\author{
G. Cremonese \\ gabriele.cremonese@inaf.it \\ D. Janches \\ diego.janches@nasa.gov
}
A.A. Berezhnoy
a_tolok@mail.ru

\author{
A.A. Christou \\ Apostolos.Christou@Armagh.ac.uk \\ T. Hirai \\ hirai.takayuki@perc.it-chiba.ac.jp \\ M. Horányi \\ mihaly.horanyi@lasp.colorado.edu
}

J.M. Jasinski

jamie.m.jasinski@jpl.nasa.gov

M. Sarantos

menelaos.sarantos-1@nasa.gov

1 ITM Physics Laboratory, NASA Goddard Space Flight Center, Code 675, 8800 Greenbelt Rd, Greenbelt MD, 20771 USA

2 Sternberg Astronomical Institute, Moscow State University, Universitetskij pr., 13, Moscow, 119234 Russia

3 Institute of Physics, Kazan Federal University, Kremlyovskaya Str., 18, Kazan, 420008 Russia

4 Armagh Observatory and Planetarium, College Hill, Armagh BT61 9DG, UK

5 INAF-Astronomical Observatory of Padova, Vicolo dell'Osservatorio 5, 35122 Padova, Italy

6 Planetary Exploration Research Center (PERC), Chiba Institute of Technology, Narashino, Tsudanuma 2-17-1, 275-0016 Chiba, Japan

7 Department of Physics, University of Colorado Boulder, 392 UCB, Boulder, CO, 80309, USA

8 Laboratory for Atmospheric and Space Physics, 1234 Innovation Dr., Boulder, CO, 80303, USA

9 Institute for Modeling Plasma, Atmospheres, and Cosmic Dust, 3400 Marine St., Boulder, CO, 80303, USA

10 NASA Jet Propulsion Laboratory, California Institute of Technology, Pasadena, CA, USA

11 Heliophysics Science Division, NASA Goddard Space Flight Center, Code 673, 8800 Greenbelt Rd, Greenbelt MD, 20771 USA 